\title{
Coherent Coding of Spatial Position Mediated by Theta Oscillations in the Hippocampus and Prefrontal Cortex
}

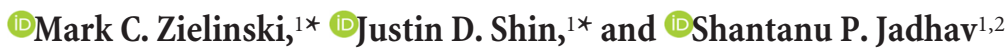 \\ ${ }^{1}$ Graduate Program in Neuroscience, and ${ }^{2}$ Neuroscience Program, Department of Psychology and Volen National Center for Complex Systems, Brandeis \\ University, Waltham, Massachusetts, 02453
}

Interactions between the hippocampus (area CA1) and prefrontal cortex (PFC) are crucial for memory-guided behavior. Theta oscillations $(\sim 8 \mathrm{~Hz})$ underlie a key physiological mechanism for mediating these coordinated interactions, and theta oscillatory coherence and phase-locked spiking in the two regions have been shown to be important for spatial memory. Hippocampal place-cell activity associated with theta oscillations encodes spatial position during behavior, and theta phase-associated spiking is known to further mediate a temporal code for space within CA1 place fields. Although prefrontal neurons are prominently phase-locked to hippocampal theta oscillations in spatial memory tasks, whether and how theta oscillations mediate processing of spatial information across these networks remains unclear. Here, we addressed these questions using simultaneous recordings of dorsal CA1-PFC ensembles and population decoding analyses in male rats performing a continuous spatial working memory task known to require hippocampal-prefrontal interactions. We found that in addition to CA1, population activity in PFC can also encode the animal's current spatial position on a theta-cycle timescale during memory-guided behavior. Coding of spatial position was coherent for CA1 and PFC ensembles, exhibiting correlated position representations within theta cycles. In addition, incorporating theta-phase information during decoding to account for thetaphase associated spiking resulted in a significant improvement in the accuracy of prefrontal spatial representations, similar to concurrent CA1 representations. These findings indicate a theta-oscillation-mediated mechanism of temporal coordination for shared processing and communication of spatial information across the two networks during spatial memory-guided behavior.

Key words: hippocampal-prefrontal synchrony; hippocampus; phase-locked spiking; prefrontal cortex; spatial memory; theta oscillations

Significance Statement

Theta oscillation- $(\sim 8 \mathrm{~Hz})$ mediated interactions between the hippocampus and prefrontal cortex are known to be important for spatial memory. Hippocampal place-cell activity associated with theta oscillations underlies a rate and temporal code for spatial position, but it is not known whether these oscillations mediate simultaneous coding of spatial information in hippocampalprefrontal networks. Here, we found that population activity in prefrontal cortex encodes animals' current position coherently with hippocampal populations on a theta-cycle timescale. Further we found that theta phase-associated spiking significantly improves prefrontal coding of spatial position, in parallel with hippocampal coding. Our findings establish that theta oscillations mediate a temporal coordination mechanism for coherent coding of spatial position in hippocampal-prefrontal networks during memory-guided behavior.

\section{Introduction}

The hippocampus and prefrontal cortex (PFC) are both necessary for learning and memory, and their interactions are critical

Received Jan. 13, 2019; revised March 5, 2019; accepted March 31, 2019.

Author contributions: M.C.Z., J.D.S., and S.P.J. designed research; M.C.Z., J.D.S., and S.P.J. performed research; M.C.Z. and S.P.J. analyzed data; M.C.Z. and S.P.J. wrote the paper.

This work was supported by NIH Grants R01 MH112661, a Sloan Research Fellowship in Neuroscience (Alfred P. Sloan Foundation), and Whitehall Foundation award to S.P.J. We thank the late John Lisman for inspiring the investigation of theta phase and prefrontal spatial representations.

The authors declare no competing financial interests.

*M.C.Z. and J.D.S. contributed equally to this work.

Correspondence should be addressed to Shantanu P. Jadhav at shantanu@brandeis.edu. for memory-guided behavior (Preston and Eichenbaum, 2013; Shin and Jadhav, 2016; Eichenbaum, 2017). Multiple inactivation studies in rodent spatial behavioral tasks have established a necessary role for functional interactions between the two regions (Floresco et al., 1997; Riedel et al., 1999; Wang and Cai, 2008; Churchwell et al., 2010; Maharjan et al., 2018). The physiological mechanisms that underlie these interactions, and how they support spatial memory processing, are thus key questions in the field. 
Theta oscillations are prominent hippocampal local field potential oscillations $(\sim 8 \mathrm{~Hz})$ associated with place-cell activity during spatial exploration in rodents, and have been established as a key physiological mechanism for mediating hippocampal (area CA1)-PFC interactions (Hyman et al., 2005; Jones and Wilson, 2005a; Siapas et al., 2005; Gordon, 2011; Shin and Jadhav, 2016). Theta-mediated CA1-PFC interactions play a crucial role in spatial memory tasks, and manifest as oscillatory coherence between theta rhythms in the two regions, as well as phase-locked spiking of neurons to hippocampal theta oscillations (Jones and Wilson, 2005a; Benchenane et al., 2010). Importantly, both oscillatory coherence and prefrontal phase-locked spiking have been shown to support performance in spatial memory tasks (Jones and Wilson, 2005a; Benchenane et al., 2010; Hyman et al., 2010; Hallock et al., 2016).

Theta oscillation-mediated hippocampal-prefrontal synchrony is therefore important for memory, but how this synchrony supports shared information processing to enable spatial memory behavior is still under investigation. There is evidence to suggest these interactions play a role in maintaining task-related representations for neurons in both regions (Jones and Wilson, 2005a; Hyman et al., 2011; Spellman et al., 2015), but several important questions about theta-mediated spatial representations remain unresolved. First, although spatial position is the most fundamental feature represented by hippocampal place-cell activity during theta oscillations (O'Keefe and Recce, 1993), whether spatial information is coherently encoded in the hippocampal-prefrontal network is not known. Spatial position during behavior can be accurately decoded from hippocampal ensemble activity (Wilson and McNaughton, 1993; Brown et al., 1998; Jensen and Lisman, 2000), and prefrontal neurons are known to have spatially selective representations (Hyman et al., 2010; Guise and Shapiro, 2017). However, no study has used simultaneous recordings and ensemble decoding to investigate whether hippocampal and prefrontal populations can coherently encode spatial position, given the evidence for strong thetamediated interactions. Second, the role of theta phase-associated PFC spiking in spatial coding is not clear. It is known that in addition to a place-cell firing rate code in CA1, theta oscillations further underlie a temporal code for space based on theta phase precession, with the timing of a CA1 spike relative to the ongoing theta phase conveying finer information about where an animal is within a place field (O'Keefe and Recce, 1993; Skaggs et al., 1996; Harris et al., 2002; Schmidt et al., 2009). This phase-based temporal code significantly improves the encoding of spatial position by CA1 ensembles when theta phase is taken into account (Jensen and Lisman, 2000). Theta phase-locking as well as theta phase precession has been reported in prefrontal neurons (Jones and Wilson, 2005a,b; Hyman et al., 2010), but whether this phase-associated spiking leads to a similar improvement in spatial coding by prefrontal ensembles is not known.

We therefore addressed these questions using simultaneous hippocampal-prefrontal ensemble recordings and decoding analyses in rats performing a spatial memory task that requires their interactions (Maharjan et al., 2018). We find that prefrontal population activity can indeed encode the animal's current position on a theta-cycle timescale coherently with CA1 coding, with correlated representations of position in the two regions. Further, we find that similar to CA1 representations, incorporation of theta phase also significantly improved prefrontal coding of spatial position, while maintaining coherent CA1-PFC coding. Our findings thus establish that theta oscillations mediate temporal

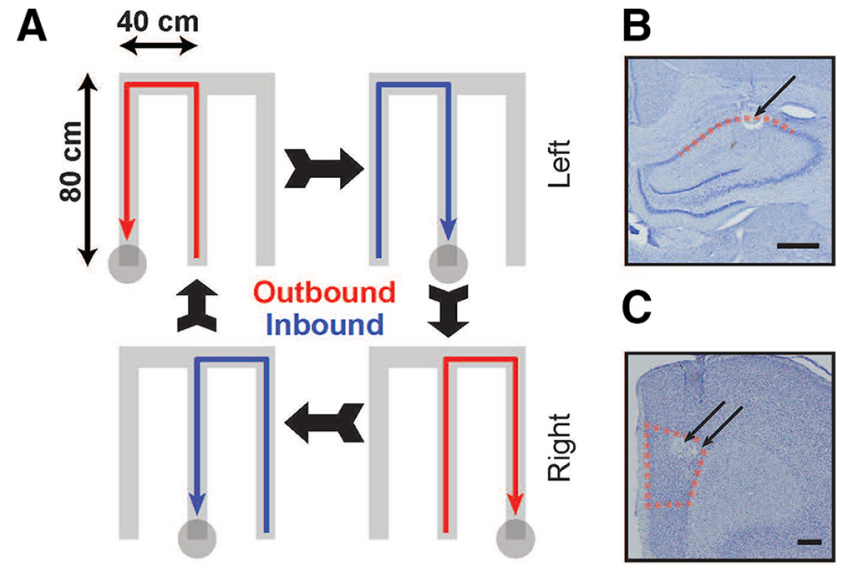

Figure 1. Experimental design and recording locations. $\boldsymbol{A}$, Schematic of W-track spatial alternation task. Animals run in a W-shaped maze, alternating between runs to the center arm and to the two alternating outer arms for liquid food reward (see Materials and Methods for more details). $\boldsymbol{B}, \boldsymbol{C}$, Representative histological images of recording sites marked by lesions (arrows) in dorsal CA1 (B) and medial PFC (PrL region; $\boldsymbol{C}$ ). Boundaries indicate extent of tetrode locations identified in dorsal CA1 and PFC in all five animals. Scale bars, $500 \mu \mathrm{m}$.

coordination of hippocampal-prefrontal activity for coherent coding of spatial position during memory-guided behavior.

\section{Materials and Methods}

Animals and experimental design. Five adult male Long-Evans rats (450550 g, 4-6 months; RRID:RGD_2308852) were used in this study. All procedures were conducted in accordance with the guidelines of the National Institutes of Health and approved by the Institutional Animal Care and Use Committee at Brandeis University. Animals were individually housed and kept on a standard $12 \mathrm{~h}$ light/dark cycle, with initial ad libitum food and water before training and experimental protocols. Experimental protocols were performed during animals' light cycles. As previously described (Jadhav et al., 2016; Tang et al., 2017), after daily habituation and handling, animals were food deprived to $85-90 \%$ of their ad libitum weight and trained to seek liquid food reward (condensed milk) alternating between ends on an elevated linear track $(80 \mathrm{~cm}$, $7 \mathrm{~cm}$ wide track sections). Animals were also exposed and habituated to an elevated rest box during this training period. After animals reached a criterion level (50 rewards in 15-20 min linear track sessions), they were taken off food restriction and subsequently, chronically implanted with a multitetrode drive (see Surgical procedures, euthanasia, and histology). Following recovery, animals were again food deprived to 85-90\% of their ad libitum weight and rehabituated to the training task described above. Animals were then trained on the W-track continuous alternation behavioral task (see Behavioral task), and electrodes were positioned appropriately.

Behavioral task. Rats performed a continuous W-track spatial alternation task that requires hippocampal-prefrontal interactions, as previously described (Jadhav et al., 2012, 2016; Tang et al., 2017; Maharjan et al., 2018). An experimental day consisted of multiple interleaved behavioral sessions on the W-track and rest box, consisting of 15-20 min sessions and 30-40 min rest periods, respectively. The $\mathrm{W}$-track $(\sim 80 \times$ $80 \mathrm{~cm}$ ) consists of elevated track sections ( $7 \mathrm{~cm}$ wide) with reward wells situated at the end of each of the three arms. The three arms were connected with two short sections ( $\sim 40 \mathrm{~cm}$ long; Fig. 1). Calibrated evaporated milk rewards were delivered automatically via infrared detectors integrated in reward wells on correct trials. Rats were tasked with learning a continuous spatial alternation strategy (starting from the center arm), alternating visits to either side well (outbound component) and the center well (inbound component; Figs. 1, 2). Incorrect alternations (visiting the same side well in consecutive outbound components; outbound error), incorrect side-to-side well visits (without visiting the center arm; inbound error), or perseverations (repeated visits to the same well just visited) were not rewarded. We used two behavioral sessions each from 
A

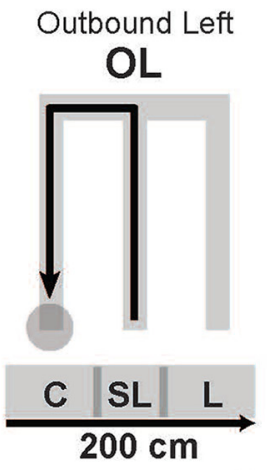

B

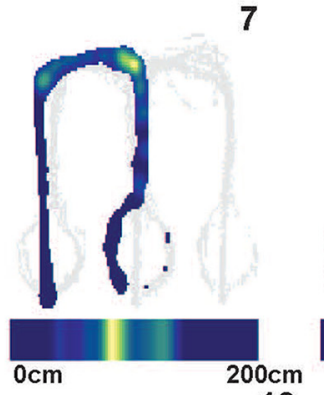

C

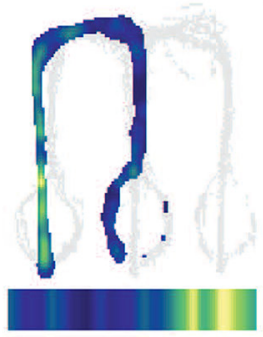

D

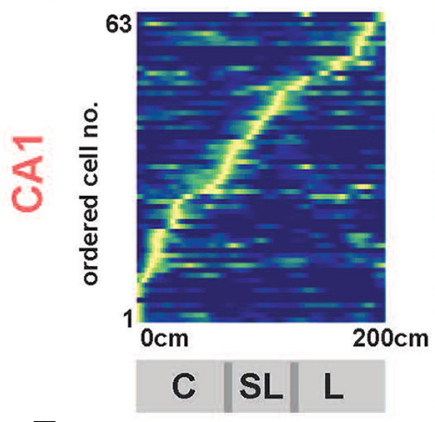

E

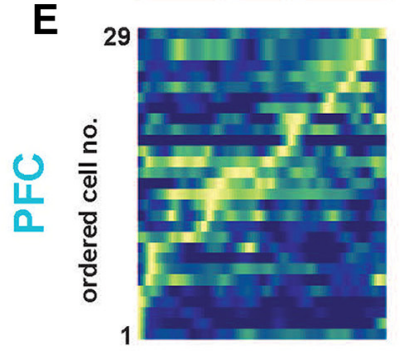

10
Inbound Left

IL

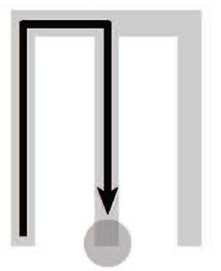

L $\quad$ SL $\quad C$
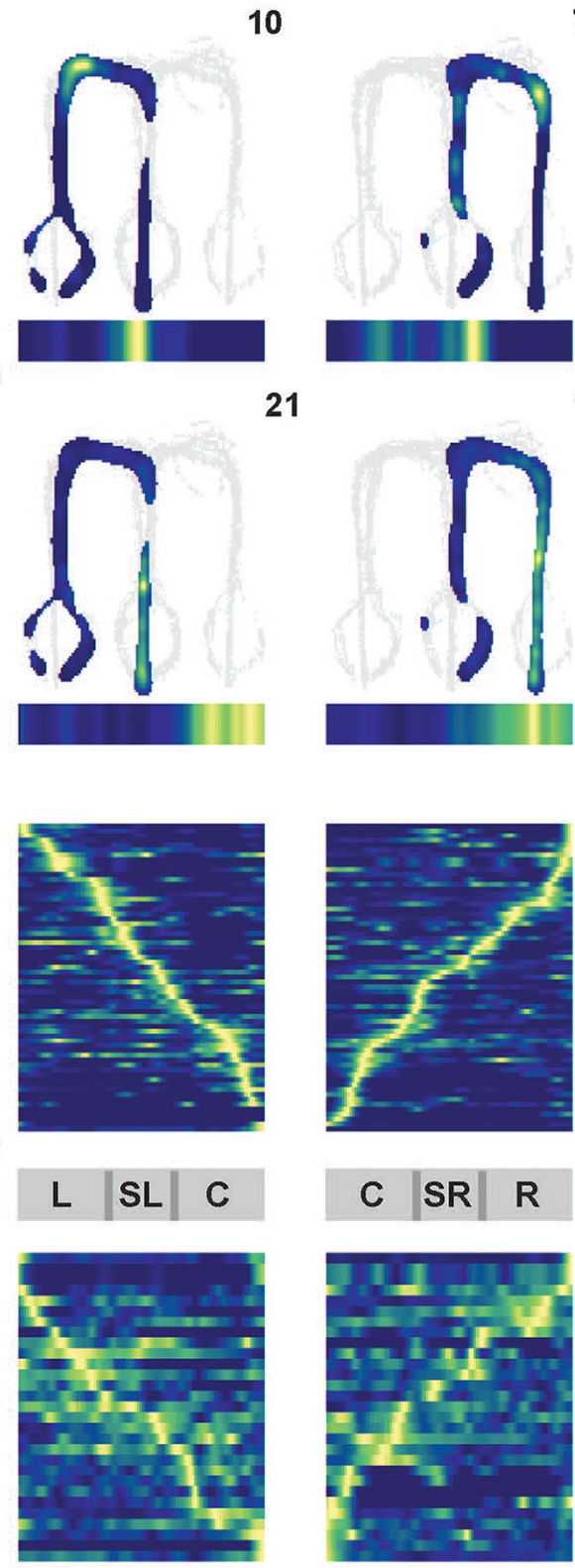

Outbound Right

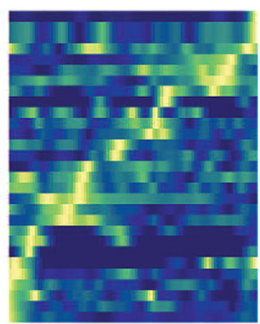

OR

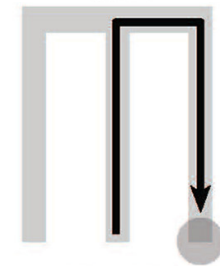

C $\quad S R \mid R$

7

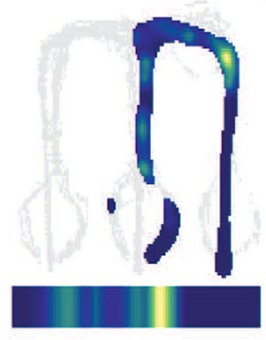

21

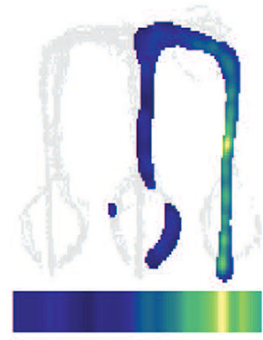

14

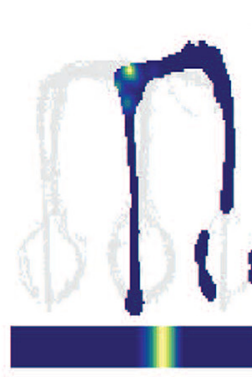

4

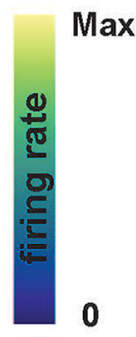

14

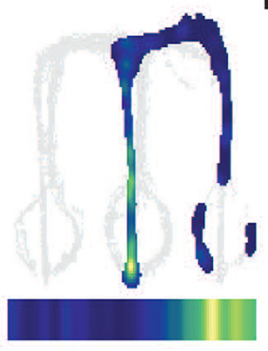

nd Rig

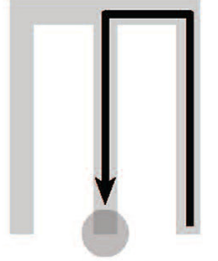

\section{R SR C}

Max

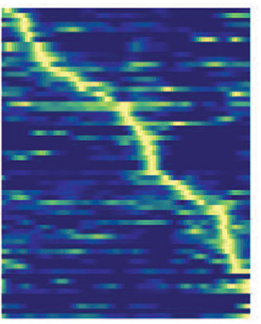

F
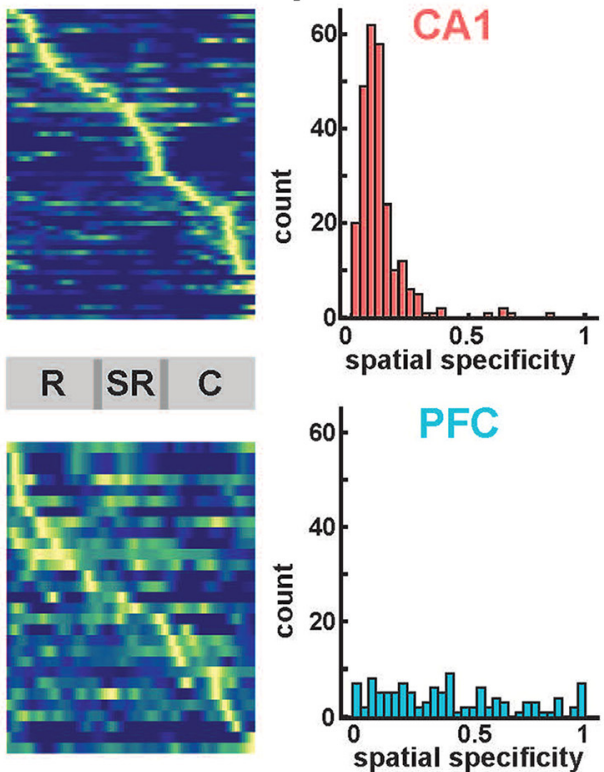

Figure 2. Spatial representation properties in CA1 and PFC. $A$, Schematic of $2 D$ firing rate maps as a function of W-track behavioral trajectories (from left to right: $O L$, IL, $O R, I R$ ). Below are schematic maps of linearized (1D) trajectories showing equivalent position with respect to the $2 D$ behavior. Track arms are labeled as described in the text (C, L, SL, R, SR). B, Occupancy normalized spatial firing rate maps of an example CA1 unit for the four separate trajectory types. Peak firing rate is reported in the top right, and linearized firing rate maps displayed below each panel. $C$, Same as in $\boldsymbol{B}$, but for a representative PFC neuron. $\boldsymbol{D}$, Population of CA1 occupancy normalized linear firing rate maps for one animal, normalized and ordered by peak firing rate for each trajectory. Population activity mapped the spatial extent in all trajectories. $\boldsymbol{E}$, Same as in $\boldsymbol{D}$, but for a representative PFC population in one animal. $\boldsymbol{F}$, Spatial specificity distributions in CA1 (top) and PFC (bottom) populations across all animals. Spatial specificity of CA1 neurons was significantly higher than PFC neurons [median spatial specificity (IQR); CA1, 0.10 (0.06-0.13); PFC: 0.37 (0.17-0.62); CA1 vs PFC: Kruskal-Wallis test, $p=1 \mathrm{e}-99]$. Detailed statistics are reported in the text. 
five animals that had learned the $\mathrm{W}$-track task and exhibited high memory performance $(87.0 \pm 2.4 \%$ for outbound task phase; $94.0 \pm 2.1 \%$ for inbound task phase). Data used for analysis was collected from the two behavioral sessions interleaved by a rest session, and neural activity was recorded continuously across the two sessions, similar to previous studies (Karlsson and Frank, 2009; Jadhav et al., 2012, 2016).

Surgical procedures, euthanasia, and histology. Surgical implantation procedures were as previously described (Jadhav et al., 2012, 2016; Tang et al., 2017), and postoperative analgesia administered for several days post-implantation. Briefly, each rat was surgically implanted with a $3 \mathrm{D}$ printed microdrive containing 30 independently moveable tetrodes, with 15 tetrodes targeting right dorsal hippocampus (AP: $-3.6 \mathrm{~mm}, \mathrm{ML}: 2.2 \mathrm{~mm}$ ) and 15 tetrodes targeting right PFC (AP: $+3.0 \mathrm{~mm}$, ML: $0.7 \mathrm{~mm}$ ). Tetrodes were made by twisting and bundling $4 \mathrm{NiCr}$ wires (diameter $13 \mu \mathrm{m}$; Sandvik Palm Coast), followed by gold electroplating to an impedance of 200-300 $\mathrm{k} \Omega$. Electrodes were gradually advanced for $2-3$ weeks following surgery to desired depths, concurrent with recovery and pretraining. The hippocampal layer was identified by characteristic LFP patterns such as presence of sharpwave ripples (SWRs), SWR polarity, and theta modulation.

At the end of the experiment, $24 \mathrm{~h}$ before euthanasia, animals were anesthetized (1-2\% isoflurane) and a current $(30 \mu \mathrm{A})$ was passed through each tetrode to form lesions at their tips for localization. Animals were later killed (Beuthanasia, $200 \mathrm{mg} / \mathrm{kg}$ ) and perfused transcardially with $4 \%$ formaldehyde using approved procedures. Brains were then fixed in $4 \%$ formaldehyde and $30 \%$ sucrose, cut into $50 \mu \mathrm{m}$ sections, stained with cresyl violet, and imaged for verification of tetrode localization.

Electrophysiology and data acquisition. All tetrodes were referenced with respect to a cerebellar ground screw. For each animal, one tetrode in corpus callosum served as hippocampal reference electrode, and another tetrode in overlying cortical regions with no spiking signals served as prefrontal reference electrode. All behavioral and electrophysiological data were acquired using a SpikeGadgets system. Digital electrophysiological data were acquired using 128 -channel digitizing head stages, sampled at $30 \mathrm{kHz}$ and saved to disk, with spike data bandpass filtered between $600 \mathrm{~Hz}$ and $6 \mathrm{kHz}$, and local field potential (LFP) bandpass filtered between $0.5 \mathrm{~Hz}$ to $400 \mathrm{~Hz}$ and downsampled to $1.5 \mathrm{kHz}$. Input and output triggers for behavioral and environmental data (e.g., reward delivery) were recorded at $1 \mathrm{~ms}$ resolution and synchronized to electrophysiological data. Animal movement and behavior was recorded and tracked using an overhead color CCD camera (30 fps), with animal head position indicated by color LEDs affixed to the headstage apparatus and microdrive. Cameras were calibrated to provide a resolution of $0.1 \mathrm{~cm} /$ pixel, and spatial extent of LEDs permitted a tracking resolution of $\sim 2 \mathrm{~cm}$.

Unit identification and inclusion. Spike peaks were identified by a threshold crossing of $40 \mu \mathrm{V}$ in the filtered spike band for CA1 and PFC, respectively. Spikes were manually sorted, and neurons were recorded and tracked continuously across the two behavior sessions, as previously described (Jadhav et al., 2012, 2016; Tang et al., 2017). Briefly, putative spikes had clustering parameters extracted (spike width on each channel, spike amplitude, and principal components), and were clustered using a custom MATLAB (MathWorks; RRID:SCR_001622) cluster visualization program (MatClust). Clusters were judged based on waveform shape, isolation distance, and lack of interspike interval violation. Only well isolated and stable putative excitatory units across the sessions were included, with putative interneurons identified and excluded based on average firing rate $\geq 15 \mathrm{~Hz}$ and spike width criteria, as previously described (Jadhav et al., 2016; Tang et al., 2017). Further, only neurons which fired at least 100 spikes in each session were included for further analysis.

Spatial maps and linearization. Two-dimensional occupancynormalized spatial firing maps were calculated for each unit when the animals' speed was $>3 \mathrm{~cm} / \mathrm{s}$, with spikes binned in $1 \mathrm{~cm}$ square bins and smoothed with a 2D Gaussian $(2 \sigma)$, excluding spiking during high ripple power ( $>3$ SD ripple band power; see LFP collection and high-theta segmentation). The linearized spiking activity of each cell was computed by assigning the rat's linear position along the $2 \mathrm{D}$ skeleton of the four possible linear behavioral trajectories (center arm reward well to outer arm reward well for outbound trajectories, and the converse for inbound trajectories; Frank et al., 2000; Jadhav et al., 2016). Spiking and occu- pancy closest to each linear $2 \mathrm{~cm}$ bin on these four trajectories was then used to calculate the smoothed, occupancy-normalized linear firing rate for correct trajectories. A peak rate of $3 \mathrm{~Hz}$ or greater was required for a cell to be considered a place cell in CA1, and a similar criterion was applied to PFC cells for inclusion in analysis (Jadhav et al., 2016). These linearized trajectories with occupancy-normalized firing rates were used in all subsequent decoding analyses.

Spatial specificity and spatial coverage. Concatenating the $1 \mathrm{D}$ firing rate maps for all four behavioral trajectories, spatial specificity was computed as the spatial sparsity of firing (Fig. 2), the proportion of firing rate bins $>25 \%$ of the peak firing rate (Jadhav et al., 2016). Spatial specificities varied from 0 for highly spatially specific neurons, to 1 for neurons with uniform firing fields (range of spatial specificity: $0-1$ ). Spatial coverage of a trajectory by an ensemble was calculated as the percentage of spatial bins with population occupancy normalized firing rate $\geq 3 \mathrm{~Hz}$ (Kay et al., 2016).

LFP collection and high-theta segmentation. LFP was bandpass filtered in the delta $(1-4 \mathrm{~Hz})$, theta $(6-12 \mathrm{~Hz})$, and ripple bands $(150-250 \mathrm{~Hz})$ using zero phase IIR Butterworth filters. We determined envelopes and phases by Hilbert transform, and took the ratio of the theta to delta envelopes at each time point for every hippocampal tetrode. High theta periods were detected using criteria for theta power, running speed, and exclusion of SWRs. Specifically, high theta windows were assigned as time periods at least one theta cycle long when the smoothed $(1 \sigma)$ mean theta/delta ratio exceeded 2, no SWRs were detected with a 3 SD threshold in the absolute power of the ripple band, and animal speed was $>3 \mathrm{~cm} / \mathrm{s}$.

Theta coherence. Coherence was calculated for each trajectory type using the Chronux (http://chronux.org/, RRID:SCR_005547) spectral processing package for MATLAB as follows. Saturating movement artifacts in the LFP were removed, and for valid time periods in which the animal's speed was $>3 \mathrm{~cm} / \mathrm{s}$, the animal's linear position was interpolated to the nearest $4 \mathrm{~cm}$ linear bin. The LFP of the PFC and CA1 tetrodes with the highest number of neurons in a given session were used to calculate coherence between the two regions. Each coherogram time bin was then assigned to a particular linear bin with the closest spatial position based on the animal's movement, leading to the average coherogram across trajectories as a function of linear distance (Fig. 3). All further analyses were averaged across animal and session for display.

Theta cycles, theta phase, and decoding. Theta LFP phase for both hippocampal and prefrontal unit spiking was relative to a hippocampal reference tetrode located in corpus callosum, as previously described (Jadhav et al., 2016). Within high theta time windows detected as described above, the troughs of the hippocampal theta-filtered LFPs were identified and used to segment valid theta cycles, discarding cycles where phase was ambiguous or reset, as previously described (Johnson and Redish, 2007; Gupta et al., 2012; Feng et al., 2015; Wu et al., 2017). Only theta cycles with at least three simultaneously active template cells in both hippocampus and prefrontal cortex were analyzed, and theta cycle decoding was implemented as previously described (Johnson and Redish, 2007; Gupta et al., 2012; Wu et al., 2017). A Bayesian decoder was used to calculate the probability of the animal's location given the firing rate templates of the neurons that fired and their spikes that occurred in each time window (Zhang et al., 1998; Davidson et al., 2009; Karlsson and Frank, 2009; Gupta et al., 2012; Pfeiffer and Foster, 2013). Briefly, the probability of the animal's position (pos) across all total spatial bins $(S)$ given a time window $(t)$, containing Poisson spiking (spikes) of independent units is as follows:

$$
P(\text { pos } \mid \text { spikes })=\frac{P(\text { spikes } \mid \text { pos }) P(\text { pos })}{P(\text { spikes })} .
$$

Normalizing over $P($ spikes $)$ and using a uniform prior $P(p o s)$ to avoid spatial decoding bias, we get the following:

$$
P(\text { pos } \mid \text { spikes })=\frac{U}{\sum_{j=1}^{S} U} \text { where } U=\left(\prod_{i=1}^{N} f_{i}(\text { pos })^{n_{i}}\right) e^{-t \sum_{i=1}^{N} f_{i}(p o s)}
$$

Where $f_{i}$ (pos) is the occupancy normalized 1D firing rate map for the $i$ th unit, $N$ is the total number of active units, $n_{i}$ is the number of spikes fired by a particular ith unit, and $t$ is a time window (in this case, the entire theta cycle). This was computed using the firing rate template for the 
Table 1. Cell counts for five animals in CA1 and PFC

\begin{tabular}{ll}
\hline CA1 & PFC \\
\hline 62 & 29 \\
53 & 25 \\
43 & 25 \\
40 & 13 \\
57 & 19 \\
\hline
\end{tabular}

corresponding behavioral trajectories. The decoded position is then the non-zero spatial bin location with the maximum posterior probability. Decoding error is defined as the absolute difference between the animal's actual position averaged in the time window and the decoded position (Fig. 3).

Cross validation metrics: firing rate templates and neuron count. Cross validation procedures were used as described previously (Fig. 3G) to generate null distributions for significance testing. To verify that the templates constructed across trials were sufficiently powered for subsequent population decoding, we used a standard leave-one- (trial) out cross validation strategy (LOOCV; van der Meer et al., 2017). Briefly, the firing of both populations during one randomly selected trajectory were left out during field/template construction, with template construction then proceeding as described above with the remaining trials. The animal position for theta cycles during this omitted trial was then decoded using these cross-validation templates, and we repeated this LOOCV procedure for all trials per session. Next, to confirm that decoding accuracy in CA1 was not simply because of a higher isolated neuron count (Fig. 3G), we confirmed our results by randomly subsetting our CA1 neuron count to match the PFC neuron count in each session and animal (number of CA1 cells was always higher than PFC cells; Table 1) and proceeding with the spatial decoding procedure as above. We repeated this procedure 1000 times per session, each with a random choice for CA1 neural subsets. For the random CA1 neuron subsets, we preserved spatial coverage and spatial specificity in CA1 (to avoid artifacts in spatial decoding because of incomplete coverage) by drawing from the CA1 neuron distribution weighted by the $20 \mathrm{~cm}$ spatial section in which their peak firing rate occurred.

Joint decoding in CA1 and PFC. Using previously described methods (Haggerty and Ji, 2015; Saleem et al., 2018), joint decoding error in each session ( $n=10$ sessions in 5 animals) was quantified as the Pearson's linear correlation coefficient between the two decoded position errors in CA1 and PFC within each time window, computed for each $20 \mathrm{~cm}$ spatial section/bin. Animal speed at each time window was classified into three evenly spaced speed bins: low, medium, and high; 3-15, 15-30, and 30+ $\mathrm{cm} / \mathrm{s}$, respectively (we obtained similar results with other thresholds for speed categories, including 3-12, 12-24, 24+ cm/s, and 3-17, 17-34, $34+\mathrm{cm} / \mathrm{s}$ ), and correlation was computed for decoding errors within each $20 \mathrm{~cm}$ spatial section/bin $(20-180 \mathrm{~cm}$ in $20 \mathrm{~cm}$ increments for each spatial bin), for subsequent comparison to shuffled controls while matching speed category and position bin. Significance for joint decoding error was computed relative to shuffled speed and position bin matched time windows, repeating the above procedure 1000 times, ensuring that correlations in decode error because of speed and location were controlled for. Joint decode errors for both real and shuffled data were binned in $1 \mathrm{~cm}^{2}$ bins and smoothed with a $2 \sigma 20 \mathrm{~cm}$ Gaussian and $z$-scored for visualization, with the residual joint decoding computed as the difference between the two (actual - shuffle; Fig. 4).

Phase locking, concentration, and precession. Phase locking (Fig. 5) was computed using a Rayleigh $z$ test for non-uniformity using the spikes of all neurons for both sessions per animal. As previously described (Jadhav et al., 2016), time periods with animal speed $>3 \mathrm{~cm} / \mathrm{s}$ and with absolute power in the ripple band $<3$ SD were considered, with spike phase derived from the hippocampal reference tetrode. Neurons with a criterion significance level of $p<0.05$ were considered significantly phase-locked. Concentration parameters $(\kappa)$ were computed for phase-locked neurons via a von Mises fit to the spiking phase data as subset above. Phase precession was computed using same criterion for neurons and spikes as above, with the addition of limiting the phase precession analysis to the peak firing field, computed as the linear distance from the peak firing rate to the first instance of firing rate $<0.25$ of the peak firing rate in both directions. Phase precession values were then computed as previously described (Kempter et al., 2012; Cei et al., 2014; Jeewajee et al., 2014; https://github.com/HoniSanders/measure_phaseprec for publicly available code). Briefly, we computed the best fit phase slope and phase intercept of a linear-circular distribution over spiking linear distance and spiking phase, respectively. Slope was limited to a maximum of one cycle over the peak firing field to reduce overfitting. All circular statistics were computed using the publicly available CircStat toolbox for MATLAB (Berens, 2009).

Theta phase splits and shuffling. For analyses involving multiple phase bins (Fig. 6), the following procedure was followed. Each theta cycle used for analysis was divided into $N$ evenly spaced subperiods divided by phase of the theta period, resulting in $N$ different spiking templates defined by theta phase for further Bayesian reconstruction. Templates were then used for every cell that corresponded to its instantaneous spike phase in theta. Shuffling protocol consisted of randomly assigning spike phase bins during template construction (encoding shuffle), or randomizing the phase bins chosen after normal template construction (decoding shuffle), and decoding normally as described above.

Data visualization. Color maps used throughout were modified from the matplotlib package and adjusted with vscim with the goal of being perceptually uniform (Thyng et al., 2016). Figure layout was generated in part using a ggplot2-like package created for MATLAB, Gramm (Morel, 2018).

\section{Results}

We used multisite multielectrode recordings to simultaneously record neural activity in dorsal CA1 region of hippocampus and medial PFC of rats $(n=5)$ as they performed a continuous W-track spatial alternation task that requires hippocampalprefrontal interactions (Kim and Frank, 2009; Jadhav et al., 2012, 2016; Tang et al., 2017; Maharjan et al., 2018). Figure 1 illustrates a schematic of the task and histologically verified recording locations in CA1 and PFC. Recordings in all animals were localized primarily to dorsal CA1 region and the prelimbic (PrL) area of PFC, delineated in Figure $1, B$ and $C$. The W-track alternation task (Fig. 1A) requires animals to visit the two outer arms of the maze in an alternating sequence, with rewarded visits from the center arm to alternating outer arms ( 2 outbound trajectories) interleaved with rewarded returns from the outer arms to the starting center arm (2 inbound trajectories; see Materials and Methods). Data from two behavior sessions per animal were used for analyses ( 2 behavior sessions interleaved by a rest session; see Materials and Methods), with animals performing the task with high accuracy in each session ( $n=10$ sessions; $n=32.6 \pm 2.8$ correct trials per session, performance: outbound task phase, mean \pm SEM; $87.0 \pm 2.4 \%$; inbound task phase; $94.0 \pm 2.1 \%$ inbound task phase). A total of 255 CA1 (animal mean \pm SEM; $51 \pm 4$ ) and 111 PFC (animal mean \pm SEM; $22 \pm 3$ ) neurons were used after exclusion of putative interneurons and those with $<100$ spikes (Table 1 shows distribution of neurons across the 5 animals; neurons were recorded continuously across the two behavior sessions for each animal, as previously described; Jadhav et al., 2012, 2016; Tang et al., 2017).

\section{Spatial representations in CA1 and PFC}

Similar to CA1 place cells, PFC neurons are also reported to have spatially restricted, trajectory-specific firing in spatial choice tasks (Jones and Wilson, 2005a; Fujisawa et al., 2008; Hyman et al., 2010; Ito et al., 2015; Jadhav et al., 2016; Guise and Shapiro, 2017; Mashhoori et al., 2018). Figure $2 A-C$ shows illustrative $\mathrm{CA} 1$ and PFC neurons with spatially specific firing fields on the four trajectories of the $\mathrm{W}$-maze task, namely, outbound to left 
arm (OL), inbound from left arm (IL), outbound from right arm (OR), inbound from right arm (IR). The four trajectories in the task are illustrated in Figure $2 A$, with the maze divided into central arm (C), left arm (L), right arm (R), and two short sections connecting the arms, side-left (SL) and side-right (SR). Each trajectory consists of a run through from one reward well to another through three sections of the maze, e.g., outbound left (OL) through center, side-left, and left arm. Spatial firing fields for each CA1 and PFC neuron were computed as occupancynormalized firing rates for each trajectory type, and then subsequently linearized for elapsed trajectory distance, similar to previous studies (Frank et al., 2000; Jadhav et al., 2016; Fig. 2 B, C, bottom shows linearized fields with a $2 \mathrm{~cm}$ bin size; see Materials and Methods for details).

Because CA1 and PFC neurons have trajectory-specific firing fields (McNaughton et al., 1983; Frank et al., 2000; Wood et al., 2000; Hok et al., 2005; Fujisawa et al., 2008; Spellman et al., 2015), each trajectory is expected to have distinct ensemble representations or spatial templates. We therefore examined the response profiles of neurons in both regions across the four behavioral trajectories (example for one animal, Fig. 2D,E). Ensemble representations in both areas tiled the full extent of the spatial environment across all trajectories, with spatial coverage sufficient for subsequent decoding analyses for all animals, calculated as the percentage of spatial bins with population occupancy normalized firing rate $\geq 3 \mathrm{~Hz}$ (Kay et al., 2016; mean percentage coverage \pm SD; CA1: $97 \pm 0.88 \%$; PFC: $100 \pm 0 \%)$. Note that neurons illustrated in Figure 2, $D$ and $E$, are sequentially ordered separately for each trajectory based on the position of their peak firing fields within that trajectory.

The spatial firing properties of CA1 and PFC were quantified by computing the spatial specificity (or, spatial sparsity) of neurons in both areas as previously described (Jadhav et al., 2016). Spatial specificity was defined as the fraction of each trajectory with above-threshold occupancy normalized firing rates $(>25 \%$ peak firing rate for each neuron; see Materials and Methods). As expected, CA1 neurons had higher spatial specificity than PFC neurons, with the CA1 distribution skewed toward lower values of spatial specificity characteristic of highly place specific cell firing, and a spread of spatial specificities in PFC (Fig. 2E; Wilcoxon signed rank test compared with uniform distributions with median $=0.5$; CA1: $Z=-13.8, p=3.1 \mathrm{e}-43$; PFC: $Z=-3.0, p=$ $0.003)$. Spatial specificity of CA1 neurons was significantly higher than PFC neurons [median spatial specificity (interquartile range [IQR]); CA1: 0.10 (0.06-0.13); PFC: 0.37 (0.17-0.62); CA1 vs PFC spatial specificity distributions are significantly different from one another, Kruskal-Wallis test; $\chi_{(1)}^{2}=101.6, p=1 \mathrm{e}-99$ ], therefore with an approximately fourfold ratio of median spatial specificities for CA1 versus PFC neurons.

\section{CA1 and PFC populations encode spatial position at a theta cycle timescale}

It is well established that theta oscillations and theta coherence are prominent in hippocampal-prefrontal networks during memory-guided trajectories (Jones and Wilson, 2005a; Siapas et al., 2005; Benchenane et al., 2010; Hyman et al., 2010; Remondes and Wilson, 2013). We similarly observed high coherence between theta oscillations for all outbound and inbound trajectories. Figure $3 A$ illustrates a coherogram exhibiting high coherence in the theta band between CA1 and PFC electrodes for a representative outbound left trajectory, and Figure $3 B$ shows average theta coherence across sessions for the four trajectories. Theta coherence was similar across trajectories (average theta coher- ence for entire trajectory; Kruskal-Wallis with Bonferroni post hoc for comparisons between the four trajectory types; $\chi_{(3)}^{2}=$ 3.88, all comparisons $p>0.63$ ).

It has been proposed that high theta coherence is suggestive of interactions on a theta oscillation timescale between hippocampal and prefrontal activity. We therefore asked, with what accuracy could we determine an animal's current position during trajectories from the concurrent neuronal ensemble activity in either area, using theta oscillations as time bins? To address this question, we used a Bayesian decoder (see Materials and Methods) to estimate the probability of an animal's position per time bin given the ensemble firing in the corresponding trajectory template, similar to previous studies (Brown et al., 1998; Jensen and Lisman, 2000).

We used the population templates in CA1 and PFC (Fig. $2 D, E)$ corresponding to an animal's current trajectory for all subsequent analyses, and corresponding theta oscillation cycles as time bins (see also Materials and Methods). Theta cycles have been proposed to represent temporal windows for organization of hippocampal-cortical activity (Itskov et al., 2008; Lisman and Redish, 2009), and previous studies have used theta cycle time bins for decoding CA1 ensemble activity (Gupta et al., 2012; Feng et al., 2015; Wang et al., 2015; Wikenheiser and Redish, 2015; Wu et al., 2017). We therefore used a similar approach for simultaneous decoding of CA1 and PFC activity. Decoded position was estimated for all four trajectories which were divided into eight 20 $\mathrm{cm}$ spatial bins or sections from the start to the end of the trajectory (Fig 3C.) We excluded the start and end sections of each arm with reward wells where animals are primarily stationary and hippocampal activity prominently exhibits SWRs (Karlsson and Frank, 2009; Jadhav et al., 2016). Using this decoding analysis approach, we asked whether both CA1 and PFC ensembles represented current spatial position on a theta time-scale, and the degree of correlation, if any, in decoding errors (as a measure of position estimate accuracy) in the two areas.

Theta cycles were extracted from filtered LFP (6-12 Hz; see Materials and Methods), and segmented based on reference cycle troughs, similar to segmentation protocols in previous studies [Johnson and Redish, 2007; Gupta et al., 2012; Feng et al., 2015; Wu et al., 2017; length of theta cycles: median (IQR), $131 \mathrm{~ms}$ (121.3-142.7 ms)]. An example of decoded positions for an outbound left trajectory, relative to actual position, using CA1 and PFC ensembles is shown in Figure 3, $D$ and $E$. The spatial bin with the highest non-zero posterior probability in a given time frame (theta cycle time) is assigned as the decoded position, and the animal's mean linearized position during the same time window is the actual position of the animal. Decoding error is reported as the difference, in $\mathrm{cm}$, between the actual and decoded position distribution of decoding error per theta cycle time bin using CA1 and PFC ensembles for all four trajectory types (OL, IL, OR, and IR) across all trials for one animal is shown in Figure $3 F(N=282$, 357,417 , and 569 time bins, respectively, in $N=10,14,12$, and 10 trials in this session for the four trajectory types; OL, IL, OR, and IR). For CA1 ensembles, the decoding error distribution is highly skewed to low values indicating high spatial decoding accuracy, as expected from previous studies [Jensen and Lisman, 2000; range of medians and IQRs for CA1 decoding error across all animals and trajectories, $2.6-4.7 \mathrm{~cm}(4.4-11.5 \mathrm{~cm})$, Shapiro-Wilk test of normality showing non-normal distributions; $p$ values for all animals and trajectories $\leq 3.4 \mathrm{e}-10$ ]. We also observed the same skewed trend toward spatial decoding accuracy in PFC [range of medians and IQRs for PFC decoding error across all animals and trajectories: $5.1-28.9 \mathrm{~cm}(12.0-59.7 \mathrm{~cm})$, Shapiro-Wilk test of 
A

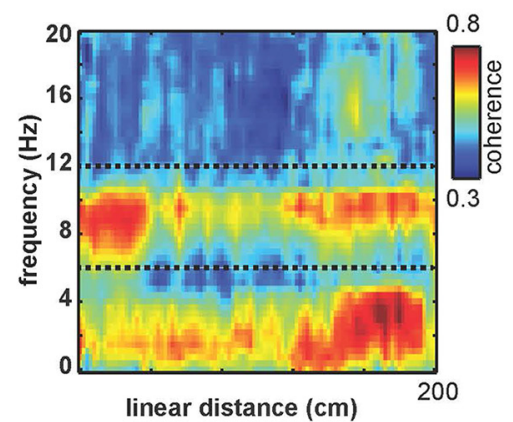

C

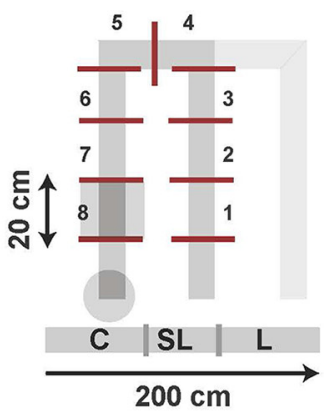

B

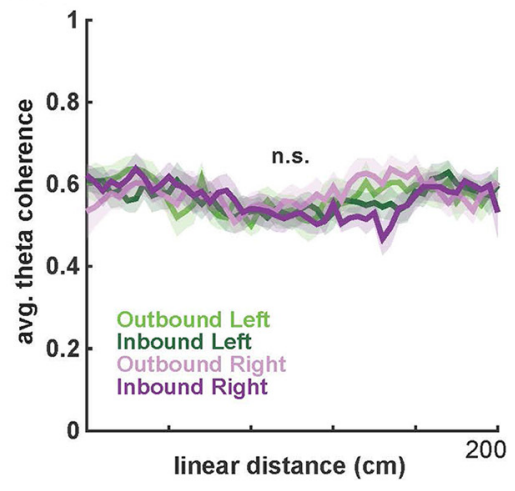

D

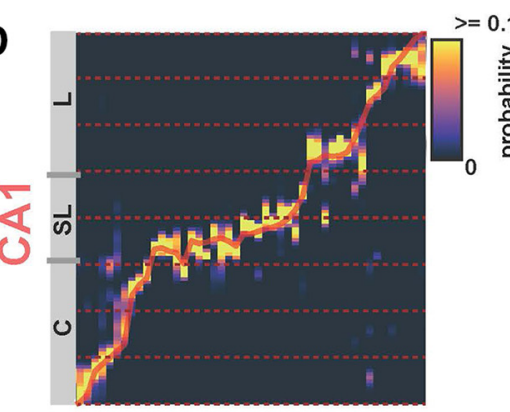

E

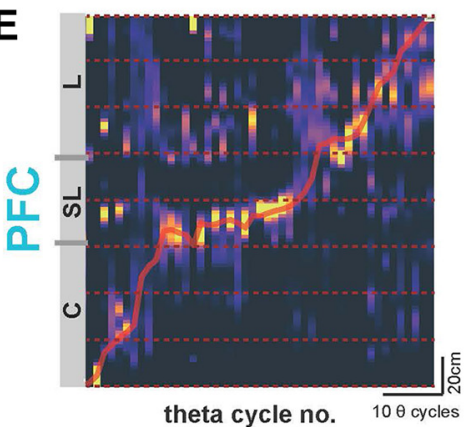

F

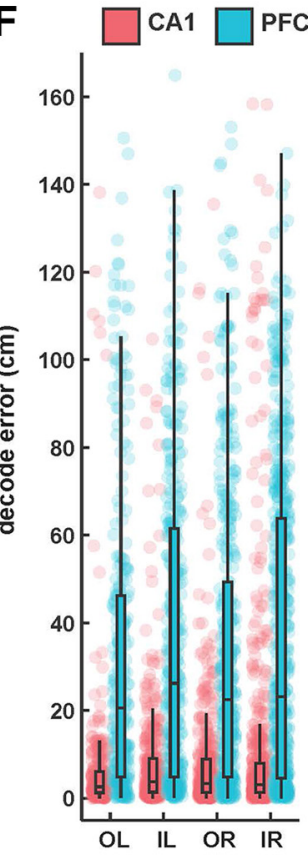

G
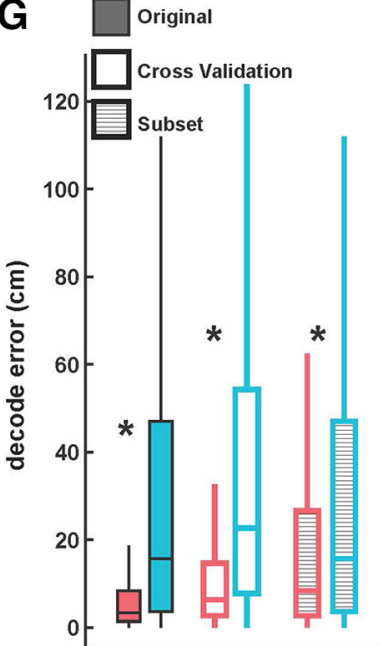

Figure 3. Decoding of spatial position from CA1 and PFC ensembles on a theta-cycle timescale. $A$, Average coherogram plotted against linear position between CA1 and PFC tetrodes for all $0 \mathrm{~L}$ trajectories across animals. Note the high coherence in the theta range throughout the memory-guided trajectory. $\boldsymbol{B}$, Average coherence in the theta band for all animals, for the four trajectory types, with SEM overlaid. Theta coherence was equivalently high for all trajectory types $(p>0.63)$. C, Schematic of spatial binning of trajectories, illustrated for $0 \mathrm{~L}$. Each trajectory was split into $20 \mathrm{~cm}$ bins (or, 8 spatial sections) for further analysis, excluding the sections with reward wells. All four trajectory types were similarly divided into eight sections. D, Actual spatial position of animal (red line) during an outbound left trajectory is shown along-with Bayesian decoded spatial position (heatmap) from CA1 ensembles ( $n=63$ neurons). Decoded position is computed as peak probability of decoded position in theta cycle time bins, using underlying theta cycles during the course of the trajectory (median length of theta cycles is $131 \mathrm{~ms}$ ). Theta cycle number is indicated along the $x$-axis, and position along the $y$-axis is shown across the eight $20 \mathrm{~cm}$ spatial sections illustrated in $\boldsymbol{C}$. $\boldsymbol{E}$, Same as in $\boldsymbol{D}$, but showing simultaneous Bayesian decoding of position using PFC ensembles ( $n=$ 29 neurons). Heatmap is similar to $\boldsymbol{D}$. F, Distributions of decoding error (difference between actual and decoded position) in theta-cycle time bins (actual values as dots, median and IQR overlaid) are shown for both CA1 and PFC ensemble decoding for all four trajectory types for one session from one animal ( $N=282,357,417$, and 569 time bins, respectively, for the four trajectory types; $0 \mathrm{~L}$, IL, OR, and IR). CA1 ensembles had significantly better decoding accuracy than PFC ensembles for all trajectory types (all $p \leq 1 \mathrm{e}-33$; detailed statistics in text). G, Comparison of CA1 and PFC decoding error under different decoding conditions, averaged across all trajectory types for all sessions. Filled bars as in $\boldsymbol{F}$; open bars using $L 00 C V$ for decoding, hatched bars using subsampling to match the number of CA1 and PFC neurons for decoding. CA1 had significantly smaller decoding error under all conditions ( ${ }^{*} p<3 e-60$; detailed statistics in the text).

normality showing non-normal distributions; $p$ values for all animals and trajectories $\leq 2.8 \mathrm{e}-08]$. Decoding error across the four trajectory types did not show differences for either CA1 or PFC populations (Kruskal-Wallis with Bonferroni correction for multiple comparisons; CA1: $\chi_{(3)}^{2}=7.04$, all pairwise comparisons $p \geq 0.05$; PFC: $\chi_{(3)}^{2}=2.55$, all pairwise comparisons $p \geq$ 0.88 ), and importantly, CA1 decoding error was significantly smaller than PFC for all four trajectory types (Wilcoxon rank sum for all trajectories; $Z \leq-12.01$, all comparisons $p \leq 1 \mathrm{e}-33$ ).
We next combined decoding errors across all trajectory types to compare and quantify CA1 and PFC decoding accuracy under different decoding conditions. Figure $3 G$ shows median decoding error using CA1 and PFC ensembles for all animals and trajectories $(N=93,61,52,68$, and 52 correct trajectories for the 5 animals over both sessions). Both CA1 and PFC activity can be used to decode animal's current position with relatively high decoding accuracy [solid bars, median (IQR); CA1: $3.4 \mathrm{~cm}(1.4-8.3$ $\mathrm{cm})$; PFC: $15.7 \mathrm{~cm}(3.7-47.0 \mathrm{~cm})]$, with significantly more accu- 
rate estimation for CA1 (CA1 vs PFC, Kruskal-Wallis, $\chi_{(1)}^{2}=$ 2504.64, $p \leq 1 \mathrm{e}-99)$. Ratio of median decoding accuracy in the two areas was comparable with the spatial specificity ratios shown in Figure 1 (median decoding error ratio for CA1 vs PFC $\sim 4.6: 1$ for these decoding parameters; spatial specificity ratio for CA1 vs PFC is $\sim 4: 1$ ). We also used a LOOCV approach for decoding, in which for decoding a given trajectory, templates used for decoding were built by omitting that trajectory (van der Meer et al., 2017; see Materials and Methods), and observed similar results [Fig. 3G, open bars; median (IQR); CA1: $6.32 \mathrm{~cm}(2.7-14.7 \mathrm{~cm})$; PFC: $22.7 \mathrm{~cm}(7.8-54.1 \mathrm{~cm})$; CA1 vs PFC, Kruskal-Wallis, $\chi_{(1)}^{2}=$ 9333.2, $p=1 \mathrm{e}^{-99}$; median decoding error ratio for CA1 vs PFC is $\sim 4: 1]$. The cross-validated decoding confirms that templates were sufficiently stable across trials to provide observed high decoding accuracy. Finally, to account for the difference in number of neurons for CA1 versus PFC, we subsampled the number of CA1 neurons to match the number of PFC neurons for each session (Fig. 3G, hatched bars; note that PFC decoding errors are same as original data with solid bars). The median decoding error ratio improved because the reduction in number of CA1 neurons increased CA1 decoding error, but the CA1 ensembles still had significantly higher decoding accuracy than PFC ensembles [Fig. $3 G$, hatched bars; median (IQR); CA1: $8.4 \mathrm{~cm}(2.7-26.6 \mathrm{~cm})$; PFC: $15.7 \mathrm{~cm}(3.7-47.0 \mathrm{~cm})$; CA1 subset vs PFC, Kruskal-Wallis, $\chi_{(1)}^{2}=272.97, p=2.6 \mathrm{e}-61$; median decoding error ratio for CA1 vs PFC is $\sim 2: 1$ ].

\section{Coherent representation of spatial position by CA1 and PFC ensembles}

To examine whether representation of spatial position is shared across hippocampus and prefrontal cortex, we first examined the effect of behavioral variables, including running speed, on decoding accuracy. Running speed is an important variable that can vary from trial-to-trial and within trajectories, and because previous results have linked theta power and spatial representations with animal running speed (McFarland et al., 1975; McNaughton et al., 1983; Geisler et al., 2007; Maurer et al., 2012), we also analyzed decoding error with regard to animal speed within a given theta window. Each theta-cycle time bin was assigned low, medium, and high speed ranges, similar to previous studies (Saleem et al., 2018; 3-15, 15-30, and $>30 \mathrm{~cm} / \mathrm{s}$ respectively; we observed similar effect for other thresholds for speed categories with boundaries at 12 and $24 \mathrm{~cm} / \mathrm{s}$, as well as 17 and $34 \mathrm{~cm} / \mathrm{s}$ ), and decoding error was compared for different speed categories. As expected, we saw a change in decoding error with speed, especially with an increase in CA1 decoding error for lower speeds (Fig. 4A; Kruskal-Wallis with Bonferroni post hoc; CA1, $\chi_{(2)}^{2}=$ $128.5, p \leq 7.8 \mathrm{e}-15$ for low vs mid and low vs high speed categories; PFC, $\chi_{(2)}^{2}=50.2, p \leq 0.008$ for low vs mid, low vs high, and mid vs high speed categories). Examining the effect of spatial bins within trajectories, we also saw a small but significant increase in decoding error at the choice point and turn sections (spatial bins 4-5 on side-left and side-right arms; Fig. $3 C$ ) corresponding to decrease in animal speed during trajectories (Fig. $4 A, C$ ). Decoding error versus spatial bin for both CA1 and PFC is shown in Figure $4 B$ (Kruskal-Wallis with Bonferroni post hoc; CA1, $\chi_{(7)}^{2}=$ 109.2, $p=1.2 \mathrm{e}-6$ for spatial Bin 5 vs spatial bin 1 ; PFC, $\chi_{(7)}^{2}=$ $44.19, p=0.03$ for spatial Bin 4 vs spatial bin 1 ), and animal speed in spatial sections during trajectories is shown in Figure $4 C$ (Kruskal-Wallis with Bonferroni post hoc; $\chi_{(7)}^{2}=2165.4, p<$ $1 e-100$ for pairwise comparisons of spatial bins 3-6 to other bins indicating significant slowdown; $p \geq 0.16$ for all spatial bin comparisons within Bins 3-6). Running speed therefore had an effect on position representations in both CA1 and PFC.

We next asked whether the theta-cycle position representations of CA1 and PFC ensembles were correlated, which would suggest that spatial information is shared across the two networks. Recent studies have found similar coherent spatial representations in CA1 and visual cortical areas (Haggerty and Ji, 2015; Saleem et al., 2018). Similar to these studies, we therefore examined the theta cycle correlations between decoding errors for CA1 and PFC estimates, while controlling for actual location and speeds. In this approach, correlation between CA1 and PFC decoding errors is computed within each spatial section (spatial sections are used to control for the relationship between speed and location during shuffling), and then compared with correlation values with shuffled data while controlling for location and speed (see also Materials and Methods), i.e., shuffled correlation values for statistical comparison are obtained by shuffling CA1 and PFC data across speed-matched theta cycles within each spatial section.

We indeed found that CA1 and PFC decoding errors within theta cycles were correlated with each other (Fig. 4D-G). The distribution of CA1 vs PFC decoding error values were distributed along the diagonal with peak $\sim 0$, but were significantly correlated, similar to previously reports for CA1 and visual cortex (Saleem et al., 2018). Figure 4D illustrates the distribution of raw correlation values for CA1 versus PFC decoding errors for one spatial section, and average for all spatial sections in Figure $4 E$ (average correlation: $r=0.10, n=10$ sessions). Crucially, to control for effects of speed modulation, we compared this actual correlation to values obtained from shuffled data, where CA1 or PFC decoding error was shuffled across time bins (theta cycles) within individual spatial sections, and within the same speed category (shuffled time bins had the same speed range: low, mid, or high, as the original time bins), thus preserving the relationship between speed and position (see also Materials and Methods; Saleem et al., 2018). Figure $4 F$ shows the difference between actual and shuffled correlations, with residual decoding errors distributed principally along the diagonal, indicating that CA 1 and PFC ensembles had correlated spatial coding. Indeed, a comparison of actual versus shuffled correlation values for all 10 behavior sessions (Fig. 4G) showed that actual decoding error correlations were significantly higher than shuffled values, with a decrease from 0.10 to 0.03 (paired-sample two-tailed $t$ test; $n=10$; significant difference between actual and shuffled correlations, $t_{(9)}=$ 3.19, $p=0.01$ ).

The cross-regional correlational values for spatial representations are similar to those observed in previous studies for CA1 and visual cortex (Haggerty and Ji, 2015; Saleem et al., 2018). Although the observed correlations are small, they establish that the spatial representations in the two regions are not independent. These results therefore suggest shared and coherent coding of spatial position in the two regions governed by theta oscillations.

\section{Theta phase modulation of CA1 and PFC activity}

In addition to coherence between theta oscillations in CA1 and PFC, spiking activity in also known to be modulated by theta phase in the two regions. Phase-locked spiking and phase precession in CA1 are well established phenomena known to result in improved spatial coding by providing a temporal code for position because of a relationship of spiking within place fields to the phase of ongoing theta oscillations (O'Keefe and Recce, 1993; 
A

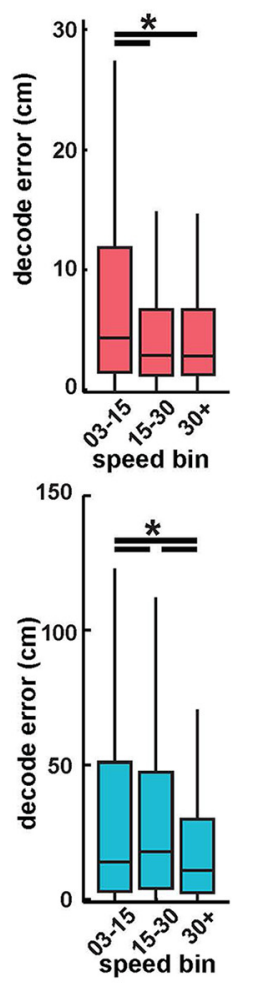

B
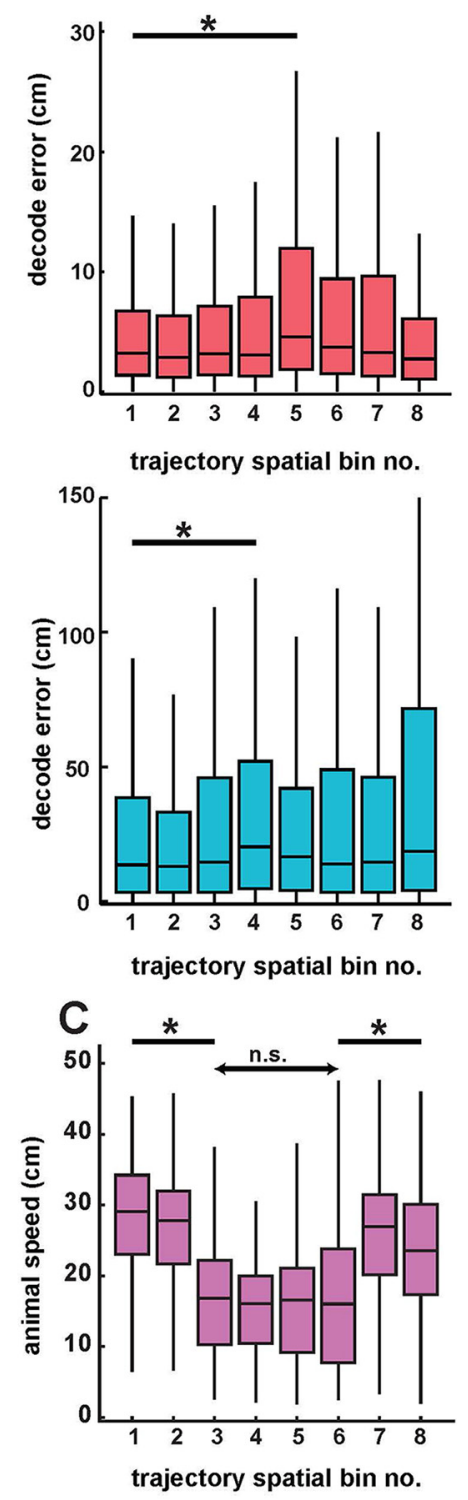

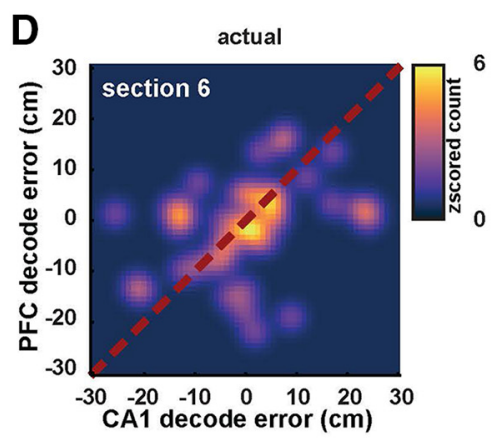

E
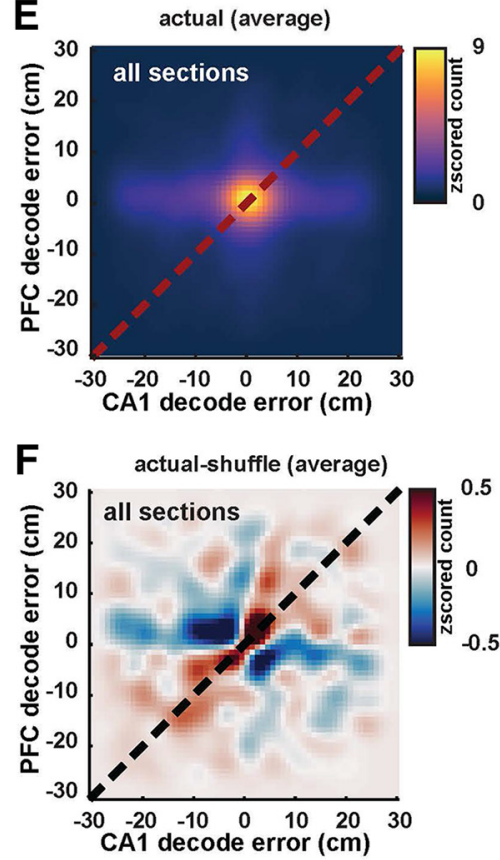

G

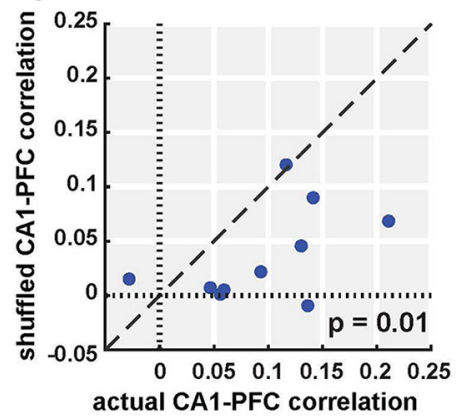

Figure 4. Coherent spatial position coding in CA1 and PFC. A, Decoding error from CA1 (pink, top) and PFC (cyan, bottom) populations in low (3-15 cm/s), medium (15-30 cm/s), and high speed $(30+\mathrm{cm} / \mathrm{s})$ categories. Decoding error varied with speed in both areas. $\boldsymbol{B}$, Decoding error as a function of spatial bin (sections) for CA1 (pink, top) and PFC (cyan, bottom). Decoding error had a small but significant increase in middle bins (Bins 4 and 5). C, Animal speed as a function of spatial bin. Animal speed decreased significantly in middle of trajectories corresponding to turn and choice point sections. D, 2D histogram showing cycle-by-cycle correlations between CA1 and PFC decoding errors in spatial section 6 . Heatmap indicates z-scored histogram count of decoding error for CA1 versus PFC. $\boldsymbol{E}$, Same as $\boldsymbol{D}$, averaged across all spatial bins. Decoding error distribution showed peaks along the diagonal $\sim 0$, with significant correlations for $C A 1$ versus $P F C$ decoding error $(r=0.10, n=$ 10 sessions, $p=0.001) . F$, Difference between decoding errors using actual and shuffled data (controlled for spatial and speed bins) shows a distribution along the diagonal, indicating strong correlations between actual decoded errors. G, Decoding error correlations for actual versus shuffled data for the 10 recording sessions. Actual correlations were significantly higher than shuffled values (mean correlation values of $r=0.10 \mathrm{vs} r=0.03, p=0.01$ ). Detailed statistics for all comparisons are reported in the text. n.s. $=$ not significant, ${ }^{*} p<0.05$ unless specified otherwise.

Skaggs et al., 1996; Harris et al., 2002; Schmidt et al., 2009; Fig. 5). Similarly, several studies have established prominent phaselocking of PFC spiking to hippocampal theta oscillations, and shown that this phase-locked spiking is correlated with performance in spatial memory tasks (Jones and Wilson, 2005a;
Benchenane et al., 2010; Spellman et al., 2015). The possibility of phase precession in PFC neurons with respect to hippocampal theta oscillations has also been reported (Jones and Wilson, 2005b). Whether this theta phase-associated spiking in PFC results in improved spatial coding is not clear. 
A
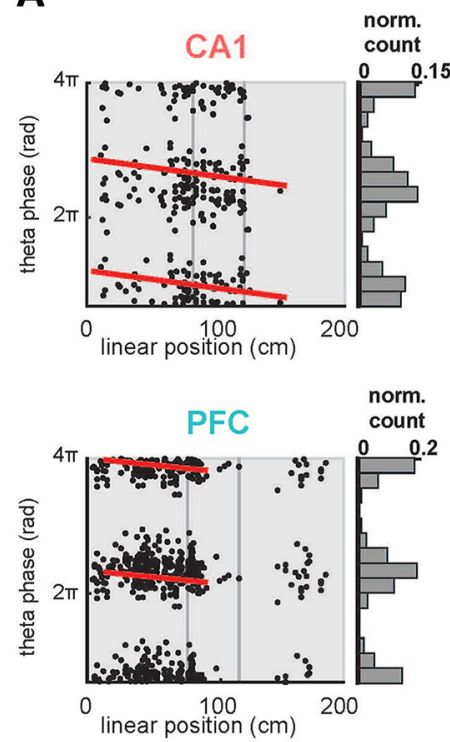

D
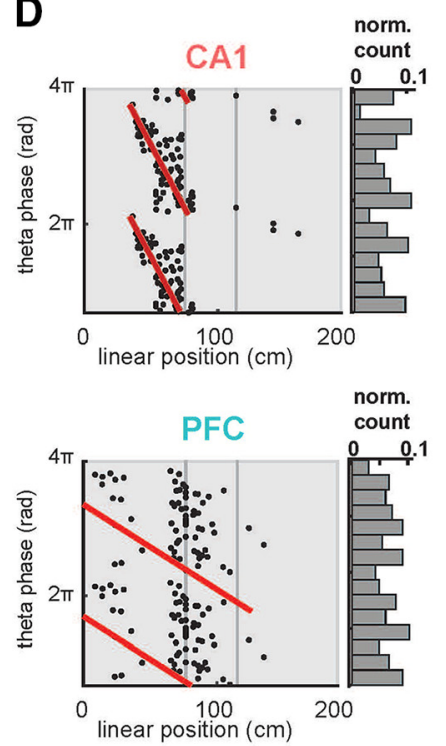

B

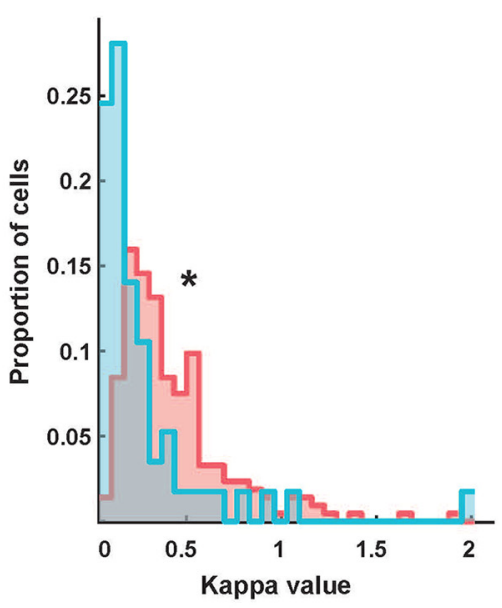

E

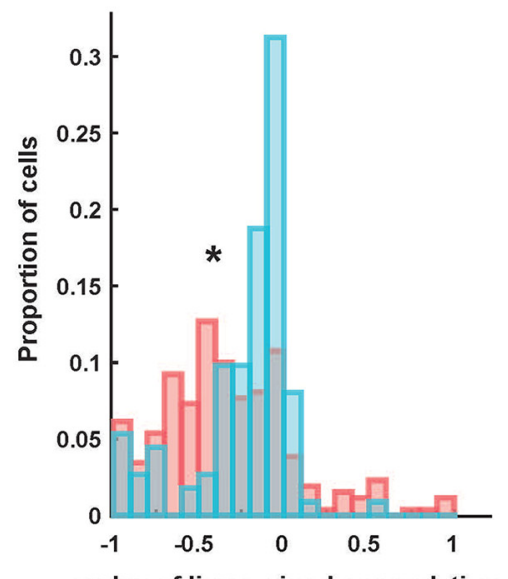

C
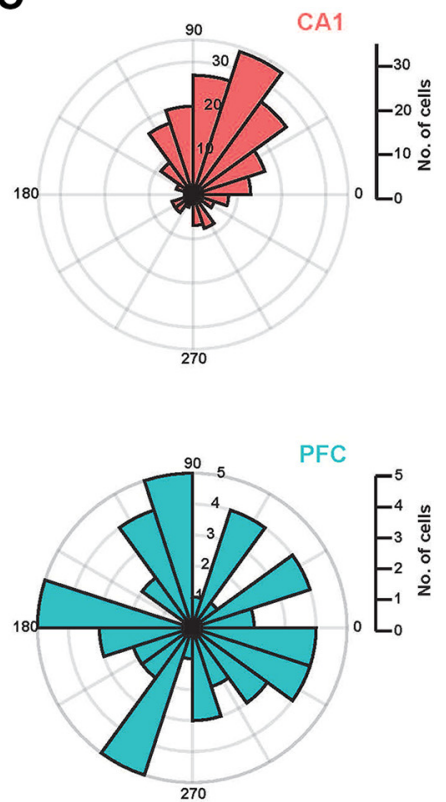

rvalue of linear-circular correlation

Figure 5. Theta phase modulation of CA1 and PFC spiking. A, Examples of theta phase-locked CA1 and PFC neurons. Spikes from single neurons are plotted with corresponding theta oscillation phase and linear position indicated along the two axes. Note that spikes occur at specific phases of theta oscillations within the firing fields of the neurons, thus exhibiting a phase-space relationship. Both neurons were significantly phase-locked, assessed with a Rayleigh ztest (CA1 neuron, $\kappa=1.1 p=1.6 \mathrm{e}-28$; PFC neuron, $\kappa=2.0, p=1 \mathrm{e}-99$ ). These neurons did not show significant phase precession ( $p>0.63$, red lines). $\boldsymbol{B}$, Distribution of $\kappa$ values for all phase-locked CA1 $(n=213)$ and PFC ( $n=57)$ neurons. CA1 had significantly stronger phase-locking than PFC $(p=1.5 \mathrm{e}-08)$. $C$, Distribution of preferred theta phases for significantly phase-locked CA1 $(n=213)$ and PFC $(n=57)$ neurons. The two distributions are significantly different from each other $(p=0.002)$. $\boldsymbol{D}$, Examples of theta phase precession in CA1 and PFC neurons. Spikes from single neurons are plotted with corresponding theta oscillation phase and linear position indicated along the two axes, with line fits for circular-linear correlation overlaid in red (CA1 neuron: $\rho=-0.64, p=1.7 \mathrm{e}-7$; PFC neuron: $\rho=-0.27, p=0.02)$. $E$, Distribution of rho value $(\rho)$ from line fits for circular-linear correlation quantifying strength of phase precession in all CA1 and PFC neurons. CA1 had significantly stronger phase precession than PFC $(p=2.7 \mathrm{e}-4) .{ }^{*} p<0.05$ unless specified otherwise.

To address this question, we first quantified theta phaseassociated spiking, namely phase-locking and phase-precession in simultaneously recorded CA1-PFC populations (see Materials and Methods; Fig. 5). Similar to previous reports by others and us (Jones and Wilson, 2005a; Siapas et al., 2005; Jadhav et al., 2016), CA1 and PFC neurons exhibited strong phase-locking, with a large fraction of neurons in both regions phase-locked to theta oscillations [Fig. 5A-C; Rayleigh $z$ test; $p<0.05$ criterion; CA1 fraction significant $=83.5 \%$ ( 213/255); PFC fraction significant $=51.4 \%(57 / 111)]$. Figure $5 A$ shows illustrative examples of significantly phase-locked CA1 and PFC neurons, along-with their spatial firing preferences on a linearized trajectory. Note that these cells exhibit both spatial selectivity as well as preferred theta phase spiking within their spatial firing fields. Spiking in the firing fields is therefore not uniformly distributed across phase, but rather occurs at particular phases of theta oscillations for phase-locked neurons. Figure $5 B$ shows distribution of phaselocking strengths for the two regions, and distribution of preferred peak phases for significantly phase-locked CA1 and PFC neurons is shown Figure 5C. As expected, phase locking strength was significantly higher for CA1 compared with PFC neurons (phase-locking strength measured as the $\kappa$ concentration param- 
eter of a circular von Mises distribution; CA1 vs PFC phaselocked neurons $n=213$ vs 57; two-sample KolmogorovSmirnov test; $p=1.5 \mathrm{e}-08$ ). Preferred phases for CA1 and PFC phase-locked neurons were also different, similar to previous reports (Kuiper two-sample test; the two distributions for preferred phases of the phase-locked neurons are significantly different, $T_{\text {(Kuiper) }}=5025, p=0.002$ ).

We next characterized phase-precession properties of CA1 and PFC neurons. Phase precession of place cells, or the advancement of firing from late to early theta phases as an animal traverses a place field, is a major constituent of CA1 theta modulation (O'Keefe and Recce, 1993; Skaggs et al., 1996; Schmidt et al., 2009; Feng et al., 2015). We quantified phase precession using a previously reported method (Kempter et al., 2012) that maximizes the circular-linear correlation of spike location versus spike theta phase, using the peak firing fields of each neuron (see also Materials and Methods). Similar to previous reports, we found a large fraction of CA1 neurons exhibited significant phase-precession (Fig. 5D,E; 58.0\% neurons, 148/255 with $p<0.05$ criterion), with a much smaller fraction of significant neurons with phase precession in PFC $(6.3 \%, 7 / 111$ with $p<$ 0.05 criterion), similar to chance levels. Illustrative examples of phase precession for CA1 and PFC are shown in Figure 5D (Fig. $5 A$ did not exhibit significant phase precession, $p>0.63$ for both neurons, CA1 neuron, $\rho=-0.64, p=1.7 \mathrm{e}-7$; PFC neuron, $\rho=$ $-0.27, p=0.02$; note that the example phase-locked neurons), and the distributions of the phase precession parameter, Rho ( $\rho$; the $R$ value equivalent for goodness-of-fit for linear circular relationships) are shown for all CA1 and PFC neurons in Figure 5E. These distributions were significantly different as expected (twosample Kolmogorov-Smirnov test; $p=2.7 \mathrm{e}-4$ ).

These results confirm previous findings of strong theta phaselocking in PFC neurons (Hyman et al., 2005; Jones and Wilson, 2005a,b; Siapas et al., 2005; Gordon, 2011; Jadhav et al., 2016). We found little evidence for PFC phase precession (Jones and Wilson, 2005b), which was similar to chance level detection rate. Interestingly, as previously suggested (Jones and Wilson, 2005a; Benchenane et al., 2010; Spellman et al., 2015), these results also show that phase-locked spiking in PFC exhibits spatial preferences, raising the possibility of a phase code for spatial location in PFC, in parallel with CA1.

\section{Incorporation of theta phase improves spatial decoding in both CA1 and PFC}

Theta phase is known to underlie an additional temporal code in CA1 that provides more accurate spatial information than just the firing rates alone, and indeed incorporation of theta phase has been shown to significantly improve hippocampal spatial decoding (Jensen and Lisman, 2000). Because PFC neurons also exhibit theta-phase-specific spatial selectivity within firing fields as a result of phase-locked spiking (Fig. 5), we next asked whether this phase relationship can lead to improved decoding accuracy in PFC populations by taking into account these phase-space relationships, in parallel with CA1 ensembles.

We used a strategy similar to a previous study (Jensen and Lisman, 2000) for incorporating theta phase in population decoding of spatial position in both CA1 and PFC, to assess and compare the impact on spatial representation in these regions (Fig. 6). Spatial firing field templates were sectioned into an increasing number of evenly spaced phase bins for decoding analyses, with each phase-bin based template taking into account theta phase in addition to the firing field of the neuron. Each neuron's firing field therefore contributes multiple templates (equivalent to number of phase bins used for decoding), which are spatially restricted only if a phase-based code for spatial position exists for the original firing field. Figure $6 \mathrm{~A}$ illustrates for a CA1 neuron how seven phase bins result in templates with spatially restricted firing because of phase precession in the firing field, with the new phase bins encoding subparts of the original firing field. Similarly, for the PFC neuron illustrated in Figure $6 B$, phase-locked spiking, and not phase precession, results in phasebased spatial templates because of spiking at specific phases within the firing field. Only the preferred phase bins (indicated with arrows) in this PFC neuron exhibits spatially localized firing. Note that for a uniform or random distribution of phases in the firing field (i.e., no phase-space relationship), multiple phase bin templates will not increase the spatial localization, but rather distribute the firing field randomly among the phase templates. Therefore, an important control for this analysis is generating a similar number of templates but with shuffled phase bins, which preserve the increased number of phase-based templates but randomize the phase-space relationship. Similarly, simply using smaller time windows without reference to phase will also not trivially increase the spatial information, because smaller time windows led to higher decoding error in both CA1 and PFC (Fig. $6 C)$, presumably because of reduction in number of spikes (Kruskal-Wallis with Bonferroni post hoc; CA1, $\chi_{(3)}^{2}=263.1, p=$ $3.7 \mathrm{e}-9$ for theta vs $50 \mathrm{~ms}$; PFC, $\chi_{(3)}^{2}=214.87, p=3.7 \mathrm{e}-9$ for theta vs $50 \mathrm{~ms}$ ).

Using this method for generating phase-based templates for population decoding, we found a significant and consistent improvement in spatial decoding accuracy in CA1 with increase in number of phase bins, similar to previous findings, with asymptotic improvement in decoding error at $\sim 6-8$ phase bins (Jensen and Lisman, 2000; Fig. 6D). There was an approximately twofold median improvement in CAl decoding accuracy when seven phase bins were taken into account [median (IQR) decode error for 1 phase bin: $3.4 \mathrm{~cm}(1.4-8.3 \mathrm{~cm})$; median (IQR) decode error for 7 phase bins: $2.0 \mathrm{~cm}(0.9-3.8 \mathrm{~cm})$; Kruskal-Wallis with Bonferroni post hoc, $\chi_{(7)}^{2}=1277.47, p \leq 0.02$ for all pairwise successive phase-bin comparisons for Bins 1-5, pairwise successive phase-bin comparisons $>5, p=1$ ]. Using a similar decoding approach for PFC ensembles, we also found a significant and strong increase in spatial decoding accuracy for PFC populations, with an approximately fourfold median improvement in PFC decoding accuracy when seven phase bins were taken into account [Fig. 6E; median (IQR) decode error for 1 phase bin: 15.7 $\mathrm{cm}(3.7-46.9 \mathrm{~cm})$; median (IQR) decode error for 7 phase bins: $3.6 \mathrm{~cm}(1.4-30.6 \mathrm{~cm})$; Kruskal-Wallis with Bonferroni post hoc, $\chi_{(7)}^{2}=1684.76, p \leq 0.0013$ for all successive pairwise comparisons for Bins 1-7, pairwise phase-bin comparison between 7 and $8, p=0.24]$. The median decoding accuracy for PFC ensembles with seven theta phase bins was comparable to the original CA1 decoding accuracy obtained with just with firing rates [median (IQR); CA1 with 1 phase bin, $3.4 \mathrm{~cm}(1.4-8.3 \mathrm{~cm})$, PFC with 7 phase bins, $3.6 \mathrm{~cm}(1.4-30.6 \mathrm{~cm})$; CA1 accuracy at 1 phase bin was still significantly higher than PFC at 7 phase bins, Wilcoxon rank sum test for comparison between the two; $z=-13.28, p=$ $3.0 \mathrm{e}-40]$.

Incorporation of theta phase thus improves spatial decoding accuracy in both CA1 and PFC, suggesting that spatial firing fields in both regions are comodulated by theta phase. To control for the possibility that the increased decoding accuracy may simply arise from increased number of templates, we performed the same analysis with shuffled firing templates, by reassigning spikes to a random theta phase bin before computing new phase-binned 
A

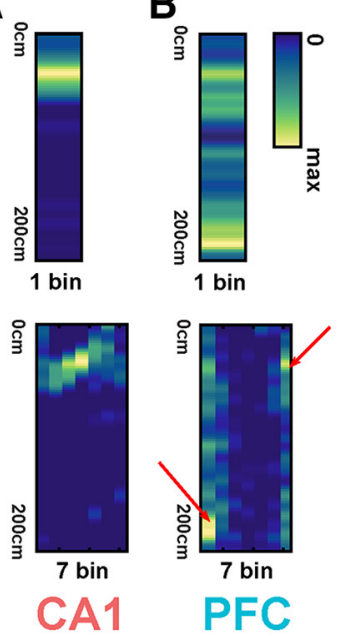

C
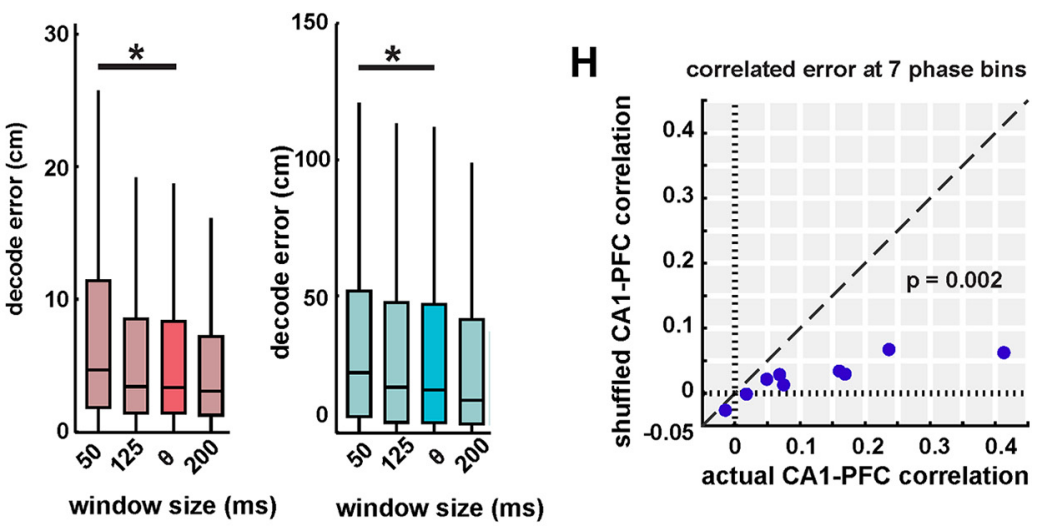

E

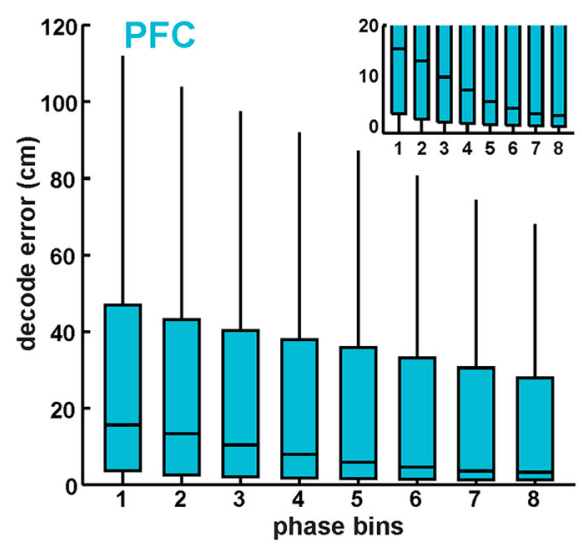

G

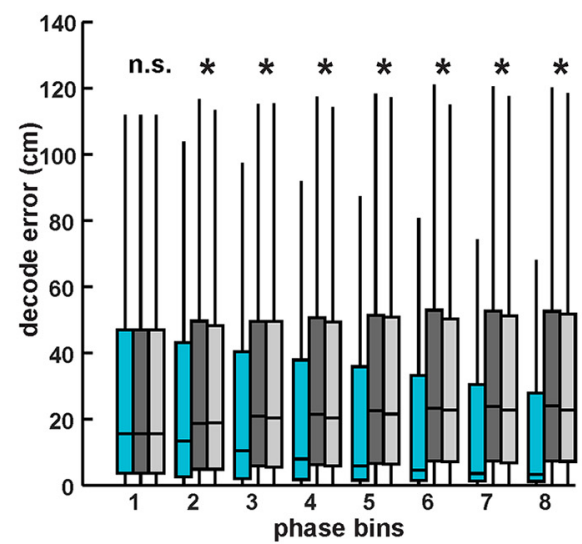

Figure 6. Theta phase-based templates improves spatial position decoding in both CA1 and PFC. $A$, Example of a CA1 firing field split into seven theta phase-based templates. This neuron exhibited strong phase precession, apparent in the phase-based templates shown on right, where preferred firing location changes with theta phase. $\boldsymbol{B}$, Example of a PFC firing field split into seven theta phase-based templates. This neuron exhibited strong phase locking, apparent in the phase-based templates shown on right, where only preferred phase bins (indicated by arrows) shows significant spiking that is spatially localized. Non-preferred phase bins exhibit highly reduced number spikes. C, Decoding error using firing rate templates as a function of time windows. Using smaller temporal windows ( 50 ms) than theta cycles leads to increased decoding error $(p<3.7 e-9)$. D, Decoding error using CA1 ensembles using successively increasing number of phase-bin templates. Phase bin 1 corresponds to a firing-rate-only template, similar to Figure 3. Decoding accuracy significantly increased until five phase bins ( $p \leq 0.02, \sim 2$-fold improvement in median decoding accuracy), with only nonsignificant, asymptomatic improvement with subsequent increase in phase bins. Inset, Low decoding error values for clarity. $\boldsymbol{E}$, Same as $\boldsymbol{D}$, using PFC ensembles. Decoding accuracy significantly increased until seven phase bins ( $p \leq 0.0013, \sim 4$-fold improvement in median decoding accuracy). $\boldsymbol{F}$, Comparison of actual decoding error for CA1 using phase-based templates to two shuffled metrics: an equivalent number of templates, but with random assignment of theta phase ("encoding" phase bin shuffle; dark gray), and with correct construction of phase templates per neuron, but with random choice of phase template during decoding (decoding phase bin shuffle; light gray). Decoding error with shuffled templates using random phase assignment did not show any significant improvement in decoding accuracy with increase in phase bins ( $p<2.3 e-114$ for all pairwise comparisons from 2 to 8 phase bins between actual and both shuffled decoding values). $\mathbf{G}$, Same as $\boldsymbol{F}$, using PFC ensembles. ( $p<3 e-34$ for all pairwise comparisons from 2 to 8 phase bins between actual and both shuffled decoding values). $\boldsymbol{H}$, Decoding error correlations for actual versus shuffled data for the 10 recording sessions for seven phase bins. Actual correlations were significantly higher than shuffled values (mean correlation values of $r=0.13$ vs $r=0.02, p=0.009)$. n.s. $=$ not significant, ${ }^{*} p<0.05$ unless specified otherwise. 
fields (encoding shuffle). This shuffling procedure thus removes any phase-space relationships by assigning spikes from firing fields to random theta phases, but preserves the original firing fields for the increased number of templates (e.g., for 7 phase bins, each of the new 7 templates will have the same overall firing field as the original field for the neuron, but with random assignment of phase bins). With these shuffled templates, we observed no improvement in decoding accuracy with increase in phase bins for either CA1 or PFC populations (Fig. 6F; pairwise Wilcoxon rank sum for encoding shuffled phase bins in CA1 2-8 vs regular phase bins $2-8, p<2.6 \mathrm{e}-208$; Fig. 6G; pairwise Wilcoxon rank sum for encoding shuffled phase bins in PFC 2-8 vs regular phase bins $2-8, p<1 \mathrm{e}-99)$. We also used another shuffling procedure, in which the construction of phase templates is preserved, but the selection of which phase templates to use during decoding is shuffled for each neuron, randomizing phase templates used during decoding ("decoding shuffle"). This decoding shuffle also did not show any improvement in decoding accuracy with increase in phase bins for either CA1 or PFC populations (Fig. 6F; pairwise Wilcoxon rank sum for decoding shuffled phase bins in CA1 2-8 vs regular phase bins $2-8, p<$ 2.3e-114; Fig. 6G; pairwise Wilcoxon rank sum for decoding shuffled phase bins in PFC 2-8 vs regular phase bins $2-8, p<$ $3 e-34)$. Thus, the preferred phase-space coding properties of multiple PFC neurons are required for the observed increase in decoding accuracy with the incorporation of theta phase.

The improved position decoding accuracy can therefore cannot be explained simply as an increase in number of templates, or the sharpening of templates because of a trivial decrease in number of spiking events in smaller time windows. Finally, we also examined whether correlations in CA1 and PFC spatial coding were preserved when theta phase was incorporated in population decoding. Using a similar approach as above (Fig. $4 F$ ), we computed correlations between actual decoding errors and shuffled errors for each session, illustrated for decoding errors obtained using seven theta phase bins (Fig. $6 H$ ). Similar to correlations based on just firing rate decoding, we found that actual decoding error correlations were significantly higher than shuffled values, with a decrease from 0.13 to 0.02 (Fig. $6 \mathrm{H}$; paired-sample twotailed $t$ test; $n=10$; significant difference between actual and shuffled correlations, $\left.t_{(9)}=3.05, p=0.009\right)$. Incorporation of theta phase thus improves spatial decoding accuracy in both CA1 and PFC.

\section{Discussion}

Our results establish a theta phase-mediated mechanism of temporal coordination for coherent coding of spatial position in hippocampal-prefrontal networks during memory-guided behavior. We report two major novel findings; first, we found that prefrontal population activity encodes animals' current position on a theta-cycle timescale during memory-guided behavior, and this prefrontal coding of spatial position is coherent with hippocampal coding. Second, we found that theta phase-associated spiking significantly improved prefrontal representations of spatial position, simultaneously with improvement in CA1 spatial representations, while maintaining coherent coding.

The physiological mechanisms that underlie hippocampalprefrontal interactions are of great interest. It is well established that these regions have complementary roles in memory processes, and multiple direct and indirect anatomical pathways between the two regions can support communication necessary for memory-guided behavior (Cenquizca and Swanson, 2007; Vertes et al., 2007; Spellman et al., 2015; Hallock et al., 2016; Maisson et al., 2018). Further, inactivation studies that target these interactions have reported deficits in spatial memory (Floresco et al., 1997; Riedel et al., 1999; Wang and Cai, 2006, 2008; Churchwell et al., 2010; Maharjan et al., 2018).

Theta oscillation-mediated interactions are the most prominently implicated physiological mechanism for long-range hippocampal-prefrontal communication in spatial memory paradigms. Theta oscillations in the two regions exhibit oscillatory coherence, and more than half of prefrontal neurons exhibit phase-locked spiking to hippocampal theta oscillations (Hyman et al., 2005; Jones and Wilson, 2005a; Siapas et al., 2005; Gordon, 2011; Guise and Shapiro, 2017). Importantly, coherence and phase-locking are enhanced during spatial memory performance (Jones and Wilson, 2005a; Benchenane et al., 2010; Hyman et al., 2010; Hallock et al., 2016; Guise and Shapiro, 2017), and impaired in parallel with cognitive deficits in genetic knock-out models (Sigurdsson et al., 2010; Harris and Gordon, 2015). Theta-mediated hippocampal-prefrontal interactions therefore support spatial memory-guided behavior, and there is evidence that these interactions can support mnemonic representations, such as task-selective activity, choice-specific responses, and memory encoding for spatial working memory (Jones and Wilson, 2005a; Hyman et al., 2011; Spellman et al., 2015; Guise and Shapiro, 2017). Despite this evidence, whether and how theta oscillations support shared processing of spatial information, the most fundamental feature represented by hippocampal place cells, has remained unexplored. Further, although theta is known to mediate a phase-based code for position in CA1, whether theta phase also influences PFC spatial coding is not clear.

In this study, we therefore had two major goals; examine spatial coding relationships in CA1 and PFC ensembles during memory-guided behavior, and investigate the effect of theta phase on spatial coding. Addressing these questions requires simultaneous population recordings in the two regions and application of Bayesian decoding analyses, which we implemented in animals performing a $\mathrm{W}$-track spatial alternation task that requires hippocampal-prefrontal interactions (Maharjan et al., 2018). We confirmed that PFC neurons exhibited spatiallyspecific firing, but as expected, CA1 place cells exhibited significantly higher spatial specificity. We then used Bayesian decoding analyses to extract moment-by-moment spatial position from ensemble activity. We used theta cycles as time bins, because (1) theta oscillations provide temporal windows for organization across hippocampal-cortical networks (Lisman, 2005; Lisman and Redish, 2009; Mizuseki et al., 2009; Lisman and Jensen, 2013), and (2) we wanted to investigate the effect of theta phase on spatial coding. For this firing rate based Bayesian decoding, using fixed time bin of lengths close to the average theta cycle $(\sim 125 \mathrm{~ms})$ yielded similar decoding error (Fig. 6), which increased with smaller time windows, presumably because of decrease in relevant spiking information.

Using this firing rate based Bayesian decoding, we found that PFC ensembles also encoded spatial position within individual theta cycles, but with higher decoding errors than CA1 as expected from their lower spatial specificity. Crucially, simultaneous population decoding allowed us to examine correlations in cycle-by-cycle decoding error. We found that CA1-PFC position representations were coherent, with decoding errors from both regions significantly correlated with each other, even when controlling for effects of speed. This coherent coding indicates shared processing of spatial signals in the CA1-PFC network, which can be supported by multiple direct and indirect connections. In particular, direct connections from ventral CA1 to PFC, as well as 
indirect connections from PFC to CA1 via nucleus reuniens, have been implicated in altering task selective spatial representations between the regions (Ito et al., 2015; Spellman et al., 2015), suggesting that a similar mechanism may underlie coherent spatial coding. These interactions can therefore be bidirectional, although we note that theta-mediated interactions have been shown to exhibit a primarily CA1-leading-PFC directionality (Gordon, 2011; Jadhav et al., 2016). Direct hippocampal-prefrontal projections arise primarily from the ventral hippocampus, which have been shown to at least partially mediate dorsal hippocampal-prefrontal interactions (O'Neill et al., 2013). Although the W-track task depends on dorsal CA1-PFC interactions, this raises the possibility that the observed theta-mediated coherent spatial coding may involve a crucial role of ventral hippocampus, which can be investigated in future studies. Interestingly, the degree of CA1-PFC spatial coherence we observed was similar in magnitude to recently reported correlations for CA1 and primary visual areas (Haggerty and Ji, 2015; Saleem et al., 2018), raising the possibility of interdependent spatial representations in widespread networks.

We next examined the effect of theta-phase associated spiking on joint spatial representations in the two regions. CA1 place cells exhibits theta phase precession, where spike timing relative to theta phase conveys finer spatial information than place field firing alone (O'Keefe and Recce, 1993; Skaggs et al., 1996). Phase precession is also related to place-cell theta sequences, which represent sequential activity within individual theta cycles that sweep through positions near the animal's current position (Foster and Wilson, 2007; Feng et al., 2015; Wikenheiser and Redish, 2015). This phase-based temporal coding results in significant improvement in estimation of position from CA1 populations (Jensen and Lisman, 2000). We therefore reasoned that PFC spatial representations may also be similarly influenced, because (1) theta oscillations mediate strong hippocampal-prefrontal interactions during memory-guided behavior, and (2) PFC neurons exhibit prominent hippocampal theta phase-associated spiking. We confirmed strong theta phase-locked spiking in both CA1 and PFC regions (Jones and Wilson, 2005a; Jadhav et al., 2016), whereas theta phase precession was prominent only in CA1 (O'Keefe and Recce, 1993; Skaggs et al., 1996). Both CA1 and PFC neurons thus exhibited a strong relationship between theta phase and spatial firing, with a preference to spike at particular phases within their spatial firing fields (CA1 by phase precession, PFC by phaselocking), underlying a phase-space relationship (Fig. 5). We asked whether using theta phase as an additional parameter in population decoding leads to an improvement in position estimation. We indeed found that both CA1 and PFC spatial representations showed significant improvement in spatial coding when theta phase was taken into account. This improvement in spatial accuracy was not seen with shuffled phase assignments, where we generated a similar number of templates, but with random assignment of phase, thus disrupting the phase-space relationship. Theta phase relationships therefore enhanced spatial accuracy, and also maintained coherent CA1-PFC spatial coding, suggesting shared spatial processing. Finally, PFC representations showed a greater improvement in spatial accuracy with phase than CA1, which was likely a floor-effect resulting from the lower limit of the spatial resolution of our position tracking $(\sim 2$ $\mathrm{cm}$ ), and the fact that CA1 firing rate representations were already highly accurate.

This increase in spatial decoding accuracy is likely because of a phase-specific segregation of spatially selective spikes from multiple neurons with similar phase preferences, leading to a sharp- ening of spatial information when decoded spikes are in the preferred phase bin. Interestingly, we observed significant improvement in decoding accuracy using up to 6-8 phase-based templates for theta cycles. It has been previously argued that for CA1, this corresponds to the phenomenon of theta-gamma coupling, where optimal improvement in decoding accuracy corresponds to number of nested gamma $(\sim 40-80 \mathrm{~Hz})$ cycles within theta cycles (Jensen and Lisman, 2000). Theta-gamma coupling is also known to be related to CA1 theta sequences (Colgin, 2011; Zheng et al., 2016), and theta-gamma coupling has also been recently reported in hippocampal-prefrontal networks (Tamura et al., 2017), raising the possibility that a similar phenomenon can influence PFC spatial representations.

Our results thus establish that hippocampal and prefrontal neural populations coherently encode position during spatial memory behavior, and further that theta phase underlies a temporal mechanism that concurrently improves spatial representational accuracy in both regions. We hypothesize that this phenomenon can possibly extend beyond local coding of current position. Interestingly, spatial decoding accuracy showed a behavioral-dependent decrease near choice points in both CA1 and PFC. Hippocampal theta sequences are known to be prominent during these behavioral epochs and support non-local representations of behaviorally relevant trajectories or upcoming goals (Johnson and Redish, 2007; Gupta et al., 2012; Wikenheiser and Redish, 2015; Zielinski et al., 2017). Our results raise the possibility that a similar mechanism of theta phase-mediated coordination can support non-local coding in hippocampal-prefrontal networks. By elucidating a physiological mechanism for coordination of spatial representations, these findings therefore provide an important foundation for future investigations of how theta oscillations organize shared mnemonic representations in hippocampal-prefrontal networks.

\section{References}

Benchenane K, Peyrache A, Khamassi M, Tierney PL, Gioanni Y, Battaglia FP, Wiener SI (2010) Coherent theta oscillations and reorganization of spike timing in the hippocampal-prefrontal network upon learning. Neuron 66:921-936.

Berens P (2009) CircStat: a MATLAB toolbox for circular statistics. J Stat Softw 31:10.

Brown EN, Frank LM, Tang D, Quirk MC, Wilson MA (1998) A statistical paradigm for neural spike train decoding applied to position prediction from ensemble firing patterns of rat hippocampal place cells. J Neurosci 18:7411-7425.

Cei A, Girardeau G, Drieu C, Kanbi KE, Zugaro M (2014) Reversed theta sequences of hippocampal cell assemblies during backward travel. Nat Neurosci 17:719-724.

Cenquizca LA, Swanson LW (2007) Spatial organization of direct hippocampal field CA1 axonal projections to the rest of the cerebral cortex. Brain Res Rev 56:1-26.

Churchwell JC, Morris AM, Musso ND, Kesner RP (2010) Prefrontal and hippocampal contributions to encoding and retrieval of spatial memory. Neurobiol Learn Mem 93:415-421.

Colgin LL (2011) Oscillations and hippocampal-prefrontal synchrony. Curr Opin Neurobiol 21:467-474.

Davidson TJ, Kloosterman F, Wilson MA (2009) Hippocampal replay of extended experience. Neuron 63:497-507.

Eichenbaum H (2017) Prefrontal-hippocampal interactions in episodic memory. Nat Rev Neurosci 18:547-558.

Feng T, Silva D, Foster DJ (2015) Dissociation between the experiencedependent development of hippocampal theta sequences and single-trial phase precession. J Neurosci 35:4890-4902.

Floresco SB, Seamans JK, Phillips AG (1997) Selective roles for hippocampal, prefrontal cortical, and ventral striatal circuits in radial-arm maze tasks with or without a delay. J Neurosci 17:1880-1890. 
Foster DJ, Wilson M (2007) Hippocampal theta sequences. Hippocampus 17:1093-1099.

Frank LM, Brown EN, Wilson MA (2000) Trajectory encoding in the hippocampus and entorhinal cortex. Neuron 27:169-178.

Fujisawa S, Amarasingham A, Harrison MT, Buzsáki G (2008) Behaviordependent short-term assembly dynamics in the medial prefrontal cortex. Nat Neurosci 11:823-833.

Geisler C, Robbe D, Zugaro M, Sirota A, Buzsáki G (2007) Hippocampal place cell assemblies are speed-controlled oscillators. Proc Natl Acad Sci U S A 104:8149-8154.

Gordon JA (2011) Oscillations and hippocampal-prefrontal synchrony. Curr Opin Neurobiol 21:486-491.

Guise KG, Shapiro ML (2017) Medial prefrontal cortex reduces memory interference by modifying hippocampal encoding. Neuron 94:183192.e8.

Gupta AS, van der Meer MA, Touretzky DS, Redish AD (2012) Segmentation of spatial experience by hippocampal theta sequences. Nat Neurosci 15:1032-1039.

Haggerty DC, Ji D (2015) Activities of visual cortical and hippocampal neurons co-fluctuate in freely moving rats during spatial behavior. eLife 4:e08902.

Hallock HL, Wang A, Griffin AL (2016) Ventral midline thalamus is critical for hippocampal-prefrontal synchrony and spatial working memory. J Neurosci 36:8372-8389.

Harris AZ, Gordon JA (2015) Long-range neural synchrony in behavior. Annu Rev Neurosci 38:171-194.

Harris KD, Henze DA, Hirase H, Leinekugel X, Dragoi G, Czurkó A, Buzsáki G (2002) Spike train dynamics predicts theta-related phase precession in hippocampal pyramidal cells. Nature 417:738-741.

Hok V, Save E, Lenck-Santini PP, Poucet B (2005) Coding for spatial goals in the prelimbic/infralimbic area of the rat frontal cortex. Proc Natl Acad Sci U S A 102:4602-4607.

Hyman JM, Zilli EA, Paley AM, Hasselmo ME (2005) Medial prefrontal cortex cells show dynamic modulation with the hippocampal theta rhythm dependent on behavior. Hippocampus 15:739-749.

Hyman JM, Zilli EA, Paley AM, Hasselmo ME (2010) Working memory performance correlates with prefrontal-hippocampal theta interactions but not with prefrontal neuron firing rates. Front Integr Neurosci 4:2.

Hyman JM, Hasselmo ME, Seamans JK (2011) What is the functional relevance of prefrontal cortex entrainment to hippocampal theta rhythms? Front Neurosci 5:24.

Ito HT, Zhang SJ, Witter MP, Moser EI, Moser MB (2015) A prefrontalthalamo-hippocampal circuit for goal-directed spatial navigation. Nature 522:50-55.

Itskov V, Pastalkova E, Mizuseki K, Buzsaki G, Harris KD (2008) Thetamediated dynamics of spatial information in hippocampus. J Neurosci 28:5959-5964.

Jadhav SP, Kemere C, German PW, Frank LM (2012) Awake hippocampal sharp-wave ripples support spatial memory. Science 336:1454-1458.

Jadhav SP, Rothschild G, Roumis DK, Frank LM (2016) Coordinated excitation and inhibition of prefrontal ensembles during awake hippocampal sharp-wave ripple events. Neuron 90:113-127.

Jeewajee A, Barry C, Douchamps V, Manson D, Lever C, Burgess N (2014) Theta phase precession of grid and place cell firing in open environments. Philos Trans R Soc Lond B Biol Sci 369:20120532.

Jensen O, Lisman JE (2000) Position reconstruction from an ensemble of hippocampal place cells: contribution of theta phase coding. J Neurophysiol 83:2602-2609.

Johnson A, Redish AD (2007) Neural ensembles in CA3 transiently encode paths forward of the animal at a decision point. J Neurosci 27:12176-12189.

Jones MW, Wilson MA (2005a) Theta rhythms coordinate hippocampalprefrontal interactions in a spatial memory task. PLoS Biol 3:e402.

Jones MW, Wilson MA (2005b) Phase precession of medial prefrontal cortical activity relative to the hippocampal theta rhythm. Hippocampus 15:867-873.

Karlsson MP, Frank LM (2009) Awake replay of remote experiences in the hippocampus. Nat Neurosci 12:913-918.

Kay K, Sosa M, Chung JE, Karlsson MP, Larkin MC, Frank LM (2016) A hippocampal network for spatial coding during immobility and sleep. Nature 531:185-190.

Kempter R, Leibold C, Buzsáki G, Diba K, Schmidt R (2012) Quantifying circular-linear associations: hippocampal phase precession. J Neurosci Methods 207:113-124.
Kim SM, Frank LM (2009) Hippocampal lesions impair rapid learning of a continuous spatial alternation task. PLoS One 4:e5494.

Lisman J (2005) The theta/gamma discrete phase code occuring during the hippocampal phase precession may be a more general brain coding scheme. Hippocampus 15:913-922.

Lisman J, Redish AD (2009) Prediction, sequences and the hippocampus. Philos Trans R Soc Lond B Biol Sci 364:1193-1201.

Lisman JE, Jensen O (2013) The theta-gamma neural code. Neuron 77: $1002-1016$.

Maharjan DM, Dai YY, Glantz EH, Jadhav SP (2018) Disruption of dorsal hippocampal-prefrontal interactions using chemogenetic inactivation impairs spatial learning. Neurobiol Learn Mem 155:351-360.

Maisson DJ, Gemzik ZM, Griffin AL (2018) Optogenetic suppression of the nucleus reuniens selectively impairs encoding during spatial working memory. Neurobiol Learn Mem 155:78-85.

Mashhoori A, Hashemnia S, McNaughton BL, Euston DR, Gruber AJ (2018) Rat anterior cingulate cortex recalls features of remote reward locations after disfavoured reinforcements. eLife 7:e29793.

Maurer AP, Burke SN, Lipa P, Skaggs WE, Barnes CA (2012) Greater running speeds result in altered hippocampal phase sequence dynamics. Hippocampus 22:737-747.

McFarland WL, Teitelbaum H, Hedges EK (1975) Relationship between hippocampal theta activity and running speed in the rat. J Comp Physiol Psychol 88:324-328.

McNaughton BL, Barnes CA, O’Keefe J (1983) The contributions of position, direction, and velocity to single unit activity in the hippocampus of freely-moving rats. Exp Brain Res 52:41-49.

Mizuseki K, Sirota A, Pastalkova E, Buzsáki G (2009) Theta oscillations provide temporal windows for local circuit computation in the entorhinalhippocampal loop. Neuron 64:267-280.

Morel P (2018) Gramm: grammar of graphics plotting in MATLAB. J Open Source Softw 3:568.

O'Keefe J, Recce ML (1993) Phase relationship between hippocampal place units and the EEG theta rhythm. Hippocampus 3:317-330.

O'Neill PK, Gordon JA, Sigurdsson T (2013) Theta oscillations in the medial prefrontal cortex are modulated by spatial working memory and synchronize with the hippocampus through its ventral subregion. J Neurosci 33:14211-14224.

Pfeiffer BE, Foster DJ (2013) Hippocampal place-cell sequences depict future paths to remembered goals. Nature 497:74-79.

Preston AR, Eichenbaum H (2013) Interplay of hippocampus and prefrontal cortex in memory. Curr Biol 23:R764-R773.

Remondes M, Wilson MA (2013) Cingulate-hippocampus coherence and trajectory coding in a sequential choice task. Neuron 80:1277-1289.

Riedel G, Micheau J, Lam AG, Roloff EL, Martin SJ, Bridge H, de Hoz L, Poeschel B, McCulloch J, Morris RG (1999) Reversible neural inactivation reveals hippocampal participation in several memory processes. Nat Neurosci 2:898-905.

Saleem AB, Diamanti EM, Fournier J, Harris KD, Carandini M (2018) Coherent encoding of subjective spatial position in visual cortex and hippocampus. Nature 562:124-127.

Schmidt R, Diba K, Leibold C, Schmitz D, Buzsáki G, Kempter R (2009) Singletrial phase precession in the hippocampus. J Neurosci 29:13232-13241.

Shin JD, Jadhav SP (2016) Multiple modes of hippocampal-prefrontal interactions in memory-guided behavior. Curr Opin Neurobiol 40:161-169.

Siapas AG, Lubenov EV, Wilson MA (2005) Prefrontal phase locking to hippocampal theta oscillations. Neuron 46:141-151.

Sigurdsson T, Stark KL, Karayiorgou M, Gogos JA, Gordon JA (2010) Impaired hippocampal-prefrontal synchrony in a genetic mouse model of schizophrenia. Nature 464:763-767.

Skaggs WE, McNaughton BL, Wilson MA, Barnes CA (1996) Theta phase precession in hippocampal neuronal populations and the compression of temporal sequences. Hippocampus 6:149-172.

Spellman T, Rigotti M, Ahmari SE, Fusi S, Gogos JA, Gordon JA (2015) Hippocampal-prefrontal input supports spatial encoding in working memory. Nature 522:309-314.

Tamura M, Spellman TJ, Rosen AM, Gogos JA, Gordon JA (2017) Hippocampal-prefrontal theta-gamma coupling during performance of a spatial working memory task. Nat Commun 8:2182.

Tang W, Shin JD, Frank LM, Jadhav SP (2017) Hippocampal-prefrontal 
reactivation during learning is stronger in awake compared with sleep states. J Neurosci 37:11789-11805.

Thyng KM, Greene CA, Hetland RD, Zimmerle HM, DiMarco SF (2016) True colors of oceanography: guidelines for effective and accurate colormap selection. Oceanography 29:9-13.

van der Meer MAA, Carey AA, Tanaka Y (2017) Optimizing for generalization in the decoding of internally generated activity in the hippocampus. Hippocampus 27:580-595.

Vertes RP, Hoover WB, Szigeti-Buck K, Leranth C (2007) Nucleus reuniens of the midline thalamus: link between the medial prefrontal cortex and the hippocampus. Brain Res Bull 71:601-609.

Wang GW, Cai JX (2006) Disconnection of the hippocampal-prefrontal cortical circuits impairs spatial working memory performance in rats. Behav Brain Res 175:329-336.

Wang GW, Cai JX (2008) Reversible disconnection of the hippocampalprelimbic cortical circuit impairs spatial learning but not passive avoidance learning in rats. Neurobiol Learn Mem 90:365-373.

Wang Y, Romani S, Lustig B, Leonardo A, Pastalkova E (2015) Theta sequences are essential for internally generated hippocampal firing fields. Nat Neurosci 18:282-288.
Wikenheiser AM, Redish AD (2015) Hippocampal theta sequences reflect current goals. Nat Neurosci 18:289-294.

Wilson MA, McNaughton BL (1993) Dynamics of the hippocampal ensemble code for space. Science 261:1055-1058.

Wood ER, Dudchenko PA, Robitsek RJ, Eichenbaum H (2000) Hippocampal neurons encode information about different types of memory episodes occurring in the same location. Neuron 27:623-633.

Wu CT, Haggerty D, Kemere C, Ji D (2017) Hippocampal awake replay in fear memory retrieval. Nat Neurosci 20:571-580.

Zhang K, Ginzburg I, McNaughton BL, Sejnowski TJ (1998) Interpreting neuronal population activity by reconstruction: unified framework with application to hippocampal place cells. J Neurophysiol 79:10171044.

Zheng C, Bieri KW, Hsiao YT, Colgin LL (2016) Spatial sequence coding differs during slow and fast gamma rhythms in the hippocampus. Neuron 89:398-408.

Zielinski MC, Tang W, Jadhav SP (2017) The role of replay and theta sequences in mediating hippocampal-prefrontal interactions for memory and cognition. Hippocampus. Advance online publication. Retrieved December 17, 2017. doi:10.1002/hipo.22821. 